УДК 519.6: 622.244: 544.012: 536.242: 532.517

\title{
МАТЕМАТИЧЕСКОЕ МОДЕЛИРОВАНИЕ И МЕТОДЫ ИССЛЕДОВАНИЯ ГИДРОДИНАМИЧЕСКОЙ ОЧИСТКИ ГОРИЗОНТАЛЬНЫХ СКВАЖИН
}

\author{
Харламов Сергей Николаевич1, \\ kharsn@mail.ru
}

\author{
Джангхорбани Мехран ${ }^{1}$, \\ mehran.janghorbani@gmail.com
}

Филиппов Константин Андреевич1, kost.filippow1@yandex.ru
1 Национальный исследовательский Томский политехнический университет, Россия, 634050, г. Томск, пр. Ленина, 30.

\begin{abstract}
Актуальность исследования определяется потребностью: уяснения современных достижений в вопросах моделирования процессов транспорта шламов в реальных условиях выполнения буровых операций, способствующих увеличению скорости бурения, повышающих эфрфективность очистки скважин; установления преимуществ/недостатков существующих подходов, современных моделей и комплексных методов описания динамики систем с частицами для эффективного воздействия на реологические свойства промывочных жидкостей; устранения причин потери оборудования.

Цель: уяснение современного опыта моделирования процессов очистки скважин со сложной образующей ее ствола, преимущественно лежащего в горизонтальной плоскости; изучение особенностей течения смесей в межтрубном пространстве $c$ круглым ядром; выдача рекомендаций в практику моделирования и расчета гидродинамических процессов, интенсифицирующих очистку.

Методы. Теоретические и практические методы исследования из смежных областей гидродинамики и тепломассопереноса в реофизически сложных системах; методы численного моделирования ламинарного и турбулентного потоков в условиях прямоточного и закрученного течений способом вращающейся стенки вокруг своей продольной оси и орбитального вращения эксиентричного ядра (внутренней трубы с подвижной/неподвижной стенкой) относительно продольной оси буровой колонны, влияющих на формирование слоя шламов и динамику частии дисперсной смеси.

Результаты. Представлены результаты современных численных исследований процессов очистки скважин с горизонтальными участками. Приведены сведения об аспектах детального гидродинамического и дифффузионного моделирования сложных течений в скважинах с криволинейной образующей. Сформулированы популярные в практике приложений модели и методы, привлекаемые для изучения характеристик течения и тепломассопереноса гомогенных и гетерогенных сред во внутренних системах. Обсуждаются аспекты моделирования процессов транспорта шламов в рамках эйлерова/лагранжева подходов с учетом особенностей, вносимых эфрфектами межфазного взаимодействия/вращения и способов закрутки. Представлены данные о деталях прогноза дисперсных систем с характеристикой влияния изменений реологических свойств (модели степенной жидкости, Гершеля-Балкли), формы/размеров частии, режимов течения смесей, инерционных сил на интенсивность образования шламов. Проанализированы проблемы повышения точности моделирования транспорта шламов, возможности новых технологий, оригинальных моделей турбулентности и их опорных двухпараметрических динамических баз, привлекаемых для интенсификации и эфрфективности процессов очистки. Даны рекомендации по их решению в рассматриваемых гидродинамических и геометрических конфигурациях. Представлены результаты и отмечены проблемы, имеющие фундаментально-прикладное значение, составляющие самостоятельный предмет перспективных исследований.
\end{abstract}

Ключевые слова:

Шлам, скважина, очистка, моделирование, гидродинамика, массообмен, реология, турбулентность, структура, силы.

\section{Введение}

В настоящее время горизонтальные скважины представляют собой наиболее распространенный тип нефтяных и газовых скважин. Однако их бурение осложнено проблемами очистки скважин от продуктов выработки. Это ставит перед специалистами задачи разработки моделей эффективности и технологий интенсификации процессов очистки с помощью данных гидродинамических исследований структуры гетерогенной смеси при сложном сдвиговом течении в рамках механики неоднородных сплошных сред, теорий сопротивления и тепломассопереноса, включающих системы полных уравнений законов сохранения массы, энергии и импульса для смеси. Однако этот путь нетривиален из-за проблем валидации гидродинамических моделей бурения и верификации их ре- зультатов особенно для турбулентных режимов, осложненных закруткой потока, а также тепло- и массопереносом при внутрифазных и межфазных взаимодействиях компонентов среды. Хорошо известно [1-3], что закрутка потока сильно интенсифицирует динамику выноса шлама из скважин, особенно в условиях добычи ископаемых ресурсов, лежащих в тонких, трещиноватых и труднодоступных залежах. Такой вид бурения существенно зависит от: точного определения траектории скважины и местонахождения долота; обеспечения устойчивости скважинных стен; эффективности очистки от бурильных продуктов.

Цель настоящей работы состоит в: уяснении современного международного опыта моделирования процессов очистки скважин со сложной образующей ее ствола и с участками, преимущественно лежащими 
в горизонтальной плоскости; изучении особенностей и закономерностей течения реологически сложной смеси в коаксиальном эксцентричном межтрубном пространстве; вылаче заключений и рекомендации в инженерную практику управления, моделирования гидродинамических и диффузионных процессов, интенсифицирующих очистку. Такие результаты представляются весьма актуальными в фундаментальном и прикладном значениях.

\section{Краткие сведения об основных принципах математического моделирования}

Как показывает анализ данных моделирования [3], экспериментальные исследования очистки рабочих областей бурового оборудования в широком диапазоне изменений локальных гидро- и газодинамических, тепло- и диффузионных параметров смеси становятся особенно эффективными в сочетании с изучением особенностей транспорта шлама методами математического моделирования на базе моделей, адекватно описывающих движение и тепломассоперенос в реологически сложной гетерогенной смеси. В такой среде процессы переноса формируются пятью группами переменных: геометрическими; гидро- и газодинамическими; тепловыми; массообменными; физикохимическими, входящими в состав математической модели, составленной из нелинейных дифференциальных уравнений в частных производных второго порядка. При этом любая (даже в значительной степени упрощенная) математическая модель, используемая для анализа столь сложных транспортных процессов, содержит некоторое количество параметров, которые должны быть определены заранее, в частности, из эксперимента. В этом смысле представленные ниже результаты важны для построения универсальных гидродинамических моделей в рамках феноменологического и статистического методов и позволяют отметить, что начало направлению поиска путей рациональной и эффективной очистки вертикальных и низко наклонных скважин положило исследование Р. Пиготта [4]. В нем установлено, что при бурении рассматриваемых скважин главным фактором является скорость скольжения смеси, при которой формируются условия осаждения частиц в ее вертикальном стволе. Отмечалось, что, если такая величина известна, следует выполнять закачку раствора при более высоких скоростях, обеспечивающих подъем шламов на поверхность. Для вычисления этой скорости следует определить силу сопротивления, тогда как остальные силы (например, гравитации и плавучести) считались известными. Дальнейшие комплексные исследования (например, [5-9]) привнесли в методики очистки ряд эмпирических связей, прогнозирующих количественные и качественные изменения интегральных параметров смеси [10]. Анализ результатов [3-10] показывает, что экспериментальный подход не лишён недостатков. Так, используемая в экспериментах установка должна быть крупномасштабной, чтобы исключить факторы воздействия на смесь, генерируемые: входными и временными эффектами (например, рис. 1, [11]); реальными реофизическими изменениями структуры бурового раствора в сравне- нии с модельным раствором из карбопола (например, [12]), часто используемым в опытах. Привлечение мощных вычислительных средств и соответствующих программных продуктов к решению указанных проблем позволяет детально исследовать пространственные гидродинамические и диффузионные эффекты в смесях.

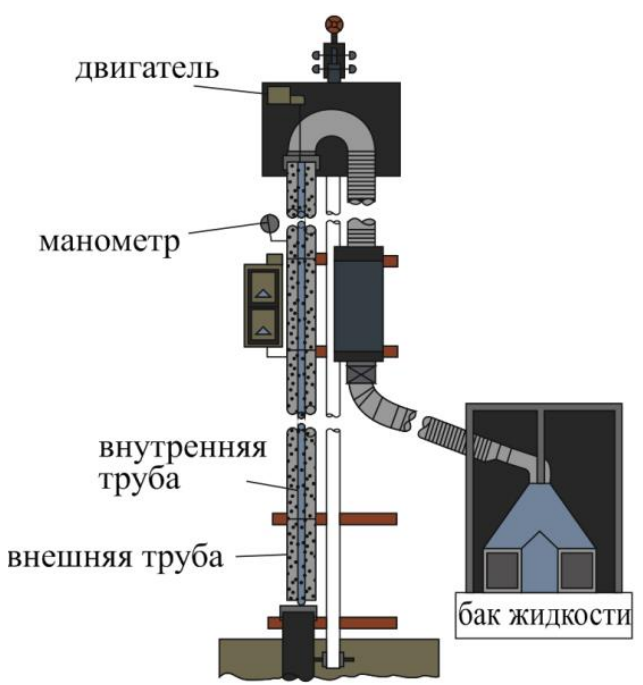

Рис. 1. Схема экспериментальной установки университета Талсы, США [11-13]

Fig. 1. Scheme of the flow loop at the university of Tulsa, USA [11-13]

Приведем некоторые полезные сведения о гидро- и газодинамических аспектах задачи, ее подходах и моделях. В частности, будем рассматривать процессы в геометрической области, сформированной конфигурацией межтрубного пространства с характерным для практики диапазоном изменений диаметров труб: внешний/внутренний диаметры $\sim(12,25 \ldots 8,5) /(5,5 \ldots 5,0)$. Заметим, что в большинстве случаев эти значения адекватны реальным размерам труб при бурении горизонтальных участков скважин. Расположение труб относительно друг друга требует определения эксцентриситета в виде (1):

$$
e=\frac{E}{R_{o}-R_{i}},
$$

где $E$ - расстояние между центрами внутренней и внешней труб с соответствующими радиусами $R_{i}, R_{o}$.

Фундаментальный анализ

Важно подчеркнуть, что в буровых операциях грязевая/буровая жидкость обладает неньютоновскими свойствами. Ее вязкость не только чувствительна к изменению полей температуры, давления, но также скорости сдвига, особенностям движения смеси по элементам оборудования. Стандартные API (American Petroleum Institute) методы для бурильной гидравлики допускают модели степенного закона и бингамовкской вязкопластической среды. Хотя в реальности большинство буровых жидкостей лучше аппроксимируются моделью Гершеля-Балкли [3] с видом связи $\mu=\Psi(\Theta)(2)$ для динамической вязкости: 


$$
\Psi(\Theta)=\left\{\begin{array}{l}
\mu_{\text {пр }}, \quad \text { при } \dot{S}<\Theta=\frac{\tau_{0}}{\mu_{\text {пр }}} ; \\
\frac{\tau_{0}+k\left(\dot{S}^{n}-\left(\frac{\tau_{0}}{\mu_{n p}}\right)^{n}\right)}{\dot{S}}, \text { при } \dot{S} \geq \Theta .
\end{array}\right.
$$

Здесь обозначено: индексом «пр» - величины, описывающие рабочий процесс до переходных эффектов в реологии; $\dot{S}$ - скорость деформаций; $\tau_{0}$ предельное напряжение; $k$ - параметр консистенции; $n$ - константа степенного закона.

Влияние геометрии частиц на течение особенно заметно в диффузионных процессах в окрестности границы формирования неподвижного слоя шламов из-за столкновений, скачков, вращающихся эффектов частиц. Об этом свидетельствуют, например, данные анализа [3]. В таких условиях предпочтительны дополнения модели связями с учетом сферичности, формы частиц [14], в виде отношения площади поверхности сферы того же объёма, что и данная частица, к площади поверхности частицы (3):

$$
\psi=\frac{\pi^{1 / 3}\left(6 V_{p}\right)^{2 / 3}}{A_{p}},
$$

где $V_{p}$ и $A_{p}$ - объём и площадь поверхности частиц соответственно.

И окончательной целью исследования является повышение значений коэффициента транспорта, который определяется как отношение (4) скорости движения шламов вдоль оси скважины к скорости потока жидкости [15]:

$$
R_{t}=\frac{V_{t}}{V_{a}}
$$

или соотношения вида (5):

$$
R_{t}=1-C
$$

где $C$ - средняя концентрация шламов в кольцевом пространстве.

Заметим, что учет различий в изменениях свойств среды, геометрии и формы транспортируемых частиц требует обоснованных заключений по упрощению физической модели объекта исследования, оценки эффектов, сопровождающих пространственное течение реологически сложной смеси в межтрубной области. В этом смысле важны предварительные исследования транспорта шламов в условиях развитой структуры несущего потока. Например, когда длина гидродинамического входного участка потока $\left(L_{h}\right)$ не попадает в область испытательной секции экспериментального оборудования, реагирующей на входные возмущения. Отдельные исследования этих вопросов показывают (например, [16-18]), что во внутренних системах с гладкими стенками величину $L_{h}$ можно определить по связи типа $L_{e n t}=f\left(D, \bar{v}, \bar{\rho}, \mu_{\text {пр }}\right)(6)$ для ламинарного и турбулентного режимов соответственHO [18]:

$$
L_{e n t}=0,062 \operatorname{Re}_{D} D ; L_{e n t}=4,4 \operatorname{Re}_{D}^{1 / 6} D .
$$

\section{Физико-математические аспекты моделирования}

Модели течений смесей включают системы взаимосвязанных дифференциальных уравнений в частных производных для законов сохранений, описывающих процессы переноса массы, импульса и тепла, с замыкающими соотношениями, характеризующими изменения локальных свойств течения, структуры потока при внутри- и межфазных трансформациях на границах раздела. Для построения численных решений таких уравнений привлекается ряд стандартных гидродинамических пакетов (FLUENT, ANSYS-CFX, STAR-CCM+ и т. д.) при допущениях, что в условиях вихревого течения в рамках RANS метода несущая фаза описывается осредненными уравнениями НавьеСтокса, замыкаемыми той или иной моделью турбулентности $[1,2,16,18])$, а также пренебрежением/учетом особенностей изменений геометрии, размеров, формы взаимодействующих частиц дисперсной фазы, особенностей их контакта на уровне «частицачастица», «частица-поверхность» стенок межтрубного пространства, заполняемого продуктами выработки.

Целесообразно кратко привести сведения о преимуществах двух популярных в расчетах течений гетерогенных сред подходов - Эйлеро-Эйлерова (ЭЭ) и Эйлеро-Лагранжева (ЭЛ). В частности, в ЭЭ подходе предполагается, что различные фазы рассматриваются как взаимодействующие и взаимопронекающие континуумы [19] в условиях, что объемная концентрация дисперсной фазы $\alpha<O\left(10^{-2}\right)$, твердые частицы представляют собой сферы заданного диаметра без деталей, определяющих их форму, а истинная плотность материала частиц значительно превосходит плотность несущей среды. В этом случае определяющие уравнения (7)-(10), характеризующие изотермическое течение смеси (твердые частицы, жидкость) согласно [19-21], имеют вид:

$$
\begin{gathered}
\frac{\partial\left(\alpha_{q} \rho_{q}\right)}{\partial t}+\nabla \cdot\left(\alpha_{q} \rho_{q} \vec{v}_{q}\right)=\sum_{p=1}^{N}\left(\dot{m}_{p q}-\dot{m}_{q p}\right)+S_{q} ;(7) \\
\frac{\partial\left(\alpha_{q} \rho_{q} \vec{v}_{q}\right)}{\partial t}+\nabla \cdot\left(\alpha_{q} \rho_{q} \vec{v}_{q} \vec{v}_{q}\right)=-\alpha_{q} \nabla p+\nabla \cdot \bar{\tau}_{q}+\alpha_{q} \rho_{q} \vec{g}+ \\
+\sum_{p=1}^{N}\left(K_{p q}\left(\vec{v}_{p}-\vec{v}_{q}\right)+\dot{m}_{p q} \vec{v}_{p q}-\dot{m}_{q p} \vec{v}_{q p}\right)+\left(\vec{F}_{q}+\vec{F}_{l i f t, q}+\vec{F}_{V m, q}\right) ;(8) \\
=\alpha_{q} \mu_{q}\left(\nabla \vec{v}_{q}+\nabla \vec{v}_{q}^{T}\right)+\alpha_{q}\left(\zeta_{q}-\frac{2}{3} \mu_{q}\right) \nabla \cdot \vec{v}_{q} \overline{\bar{I}} ;(9) \\
\frac{\partial}{\partial t}\left(\alpha_{s} \rho_{s} \vec{v}_{s}\right)+\nabla \cdot\left(\alpha_{s} \rho_{s} \vec{v}_{s} \vec{v}_{s}\right)= \\
=-\alpha_{s} \nabla p-\nabla p_{s}+\nabla \cdot \bar{\tau}_{s}+\alpha_{s} \rho_{s} \vec{g}+ \\
+\sum_{l=1}^{N}\left(K_{l s}\left(\vec{v}_{l}-\vec{v}_{s}\right)+\dot{m}_{l s} \vec{v}_{l s}-\dot{m}_{s l} \vec{v}_{s l}\right)+\left(\vec{F}_{s}+\vec{F}_{l i f t, s}+\vec{F}_{V M, s}\right) .(10)
\end{gathered}
$$

Здесь принято: «индексы» $q$ и $s$ характеризуют параметры жидкой и твердой фаз соответственно; $\rho-$ плотность; $\vec{v}$ - вектор скорости; $S_{q}-$ источниковый член; $\dot{m}_{q p}-$ интенсивность перехода массы из $p$-й в $q$ ю фазу (в нашем случае отсутствует перенос из жидкой в твердую фазу, поэтому $\left.\dot{m}_{p q}=\dot{m}_{p q}=0\right) ; \overline{\overline{\tau_{q}}}-$ тен- 
зор напряжений $q$-й фазы; $\mu_{q}, \varsigma_{q}-$ коэффициенты сдвиговой и объемной вязкостей $q$-й фазы; $p$ - давление; $K_{p q}$ - коэффициент межфазного обмена импульсом; $\vec{F}_{s}, \vec{F}_{l i f t, s}, \vec{F}_{V M, s}-$ соответственно внешняя сила, подъемная сила, дополнительная сила виртуальной массы частиц твердой фазы; $p$ - относится к давлению, используемому всеми фазами; $p_{s}$ - давление частиц твердой фазы; $K_{l s}=K_{s l}-$ коэффициент обмена импульсом между жидкой и твердой фазами. Заметим, что сила виртуальной массы заметна в анализе процессов, когда дисперсные частицы гораздо легче жидкости. Поэтому при моделировании очистки скважин ее, а также подъёмной силой (которая также мала по сравнению с силой сопротивления) можно пренебречь. Видно, что определяющее влияние на динамику оказывают: вязкие эффекты; силы градиента давления, тяжести, сопротивления, межфазных процессов.

В рамках ЭЛ подхода учитываются индивидуальные свойства частиц дисперсной фазы. При этом движение жидкой фазы описывается уравнениями Навье-Стокса подобно эйлерову подходу, динамика частиц дисперсной фазы (лагранжев подход) - уравнением движения (11) и модельными замыканиями для сил межфазного взаимодействия. Все это позволяет иметь определяющую систему уравнений законов сохранения массы и импульса жидкой фазы в виде [19-21]:

$$
\frac{\partial\left(\alpha \rho_{f}\right)}{\partial t}+\nabla \cdot\left(\alpha \rho_{f} \vec{v}_{f}\right)=0
$$

$$
\frac{\partial\left(\alpha \rho_{f} \vec{v}_{f}\right)}{\partial t}+\nabla\left(\alpha \rho_{f} \vec{v}_{f} \vec{v}_{f}\right)=-\alpha \nabla p+\alpha \nabla \bar{\tau}-\vec{S}_{f}+\alpha \rho_{f} \vec{g} .
$$

Здесь $\rho_{f}$ - плотность жидкости; $\vec{v}_{f}-$ скорость жидкости; $\alpha$ - объемная доля жидкой фазы; $\bar{\tau}-$ тензор вязких напряжений; $\vec{S}_{f}=\frac{\sum_{i=1}^{M} \vec{F}_{f, i}}{V_{\text {яч }}}-$ источниковый член спектра сил межфазного взаимодействия, влияющих на все частицы, находящиеся в данный момент в ячейке, нормированных на ее объем; М - общее число частиц дисперсной фазы в ячейке.

Данные уравнения дополняются уравнением движения частиц дисперсной фазы, вид которого и его замыкания изложены ниже.

\section{Формализм моделирования движений частиц} с произвольной геометрией

В рамках идей ЭЛ подхода (например, [17, 20, 22-31]) движение частиц в смеси под действием спектра внешних и поверхностных сил (например, тяжести, взаимодействий частиц между собой/со стенками буровой колонны/бурильной трубы, а также силами внутри- и межфазного взаимодействия, сопротивления, подъемной силы с поперечными и вращательными эффектами) можно описать уравнением транспорта частицы $p$ в потоке жидкой фазы в виде (11):

$m_{p} \frac{\partial \vec{u}_{p}}{\partial t}=m_{p} \vec{g}\left(1-\frac{\rho_{f}}{\rho_{p}}\right)+\sum_{i=1}^{k_{c}} \vec{F}_{c, i}^{p}+\vec{F}_{D}+\vec{F}_{S}+\vec{F}_{M}+\vec{F}_{p}$.
Здесь принято: $m_{p}, \rho_{p}$ - масса и плотность частицы; $\vec{F}_{c, i}^{p}$ - сила воздействия на $i$-й внешний сферический элемент частицы $p$, где $k_{c}$ отвечает числу сферических элементов на внешней поверхности каждой частицы с заданием ее физических и геометрических особенностей; $\vec{F}_{D}$ - гидродинамическая сила сопротивления; $\vec{F}_{S}$ - подъемная сила с поперечным эффектом; $\vec{F}_{M}-$ вращательная подъемная сила, или сила Магнуса; $\vec{F}_{p}-$ сила гидродинамического градиента давления. При этом, согласно [26], вращательное движение частицы $p$ можно представить в виде (12):

$$
\frac{d\left(I_{p} \vec{\omega}_{p}\right)}{d t}=\sum_{e=1}^{k_{c}}\left(\vec{T}_{t, e}^{p}+\vec{T}_{r, e}^{p}\right)+\vec{T}_{D T}^{p},
$$

где $\vec{T}_{t, e}^{p}, \vec{T}_{r, e}^{p}$ - вращательные вектора, обусловленные соответственно тангенциальной и нормальной составляющими контактной силы, действующими на отдельный элемент частицы $p$, который имеет момент инерции $I_{p}$, вращательную скорость $\vec{\omega}_{p}$ и $\vec{T}_{D T}^{p}-$ вращательное сопротивление, вызванное закрученным движением.

Особенности замыканий для взаимодействуюших сил и моментов закрутки. Обобщая опыт исследований смесей [27], целесообразно комбинацию сил в точке взаимодействия поверхностей $i$-го элемента частиц $p$ и $j$-го элемента частиц $q$ представить в виде (13):

$$
\vec{F}_{c, i}^{p}=\vec{F}_{n, i j}+\vec{F}_{n, i}^{d}+\vec{F}_{t, i j}+\vec{F}_{t, i j}^{d},
$$

где силу взаимодействия в нормальной плоскости в точке контакта поверхностей $\left(\vec{F}_{n, i j}\right)$ записывают в виде (14):

$$
\vec{F}_{n, i j}=\frac{4}{3} E^{*}\left(R^{*}\right)^{0,5} \delta_{n, i j}^{1,5},
$$

где $\delta_{n, i j}$ - символ Кронекера, учитывающий совпадения в плоскости; $E^{*}$ - эквивалентный модуль Юнга $\left(E^{*}=\left[\frac{\left(1-v_{i}^{2}\right)}{E_{i}}+\frac{\left(1-v_{j}^{2}\right)}{E_{j}}\right]^{-1}\right) ; R^{*}-$ эквивалентный радиус $\left(R^{*}=\left(\frac{2}{d_{p}}+\frac{2}{d_{q}}\right)^{-1}\right) ; E_{i}, v_{i} d_{i}, E_{j}, v_{j} d_{j}-$ соответственно параметры (модуль Юнга, коэффициент Пуассона, размеры частиц) $i$-го и $j$-го элементов частиц $p, q$.

Сила демпфирования в точке взаимодействия поверхностей $i$-го элемента частиц $p$ и $j$-го элемента частиц $q$ имеет вид (15):

$$
\vec{F}_{n, i j}^{d}=\hat{c} \frac{\ln e}{\left((\ln e)^{2}+\pi^{2}\right)^{0,5}}\left(S_{n, i j} m^{*}\right)^{0,5} \vec{v}_{n, p q},
$$

где $\hat{c} \simeq-1,826$ по [27]; $m^{*}$ - эквивалентная масса частиц $\left(m^{*}=\left(\frac{1}{m_{i}}+\frac{1}{m_{j}}\right)^{-1}\right) ; m_{i}, m_{j}-$ соответственно массы $i$-го и $j$-го элемента частиц в момент взаимодействия); $S_{n, i j}$ - параметр нормальной жесткости 
$\left(S_{n, i j}=2 E^{*}\left(R^{*} \delta_{n, i j}\right)^{0,5}\right) ; \vec{v}_{n, p q}-$ нормальная компонента относительной скорости в точке контакта; $e$ - коэффициент восстановления.

Тангенциальная компонента взаимодействующей силы [27] имеет вид (16):

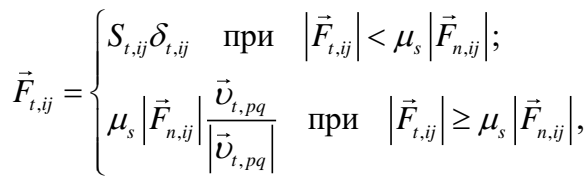

где $S_{t, i j}-$ параметр тангенциальной жесткости $\left(S_{t, i j}=8 G^{*}\left(R^{*} \delta_{t, i j}\right)^{0,5}\right) ; G^{*}$ - эквивалентный модуль сдвига; $\delta_{t, i j}$ - символ Кронекера, учитывающий совпадения в соответствующей плоскости; $\mu_{s}-$ коэффициент трения скольжения; $\vec{v}_{t, p q}$ - относительная тангенциальная скорость в точке контакта.

Тангенциальная демпфирующая сила принимается в виде (17):

$$
\vec{F}_{t, i j}^{d}=\hat{c} \frac{\ln e}{\left((\ln e)^{2}+\pi^{2}\right)^{0,5}}\left(S_{t, i j} m^{*}\right)^{0,5} \vec{v}_{t, p q} .
$$

Согласно [26] тангенциальный крутящий момент, действующий на $i$-й элемент частицы $p$ в силу столкновений с $j$-м элементом частицы $q$, имеет вид (18):

$$
\vec{T}_{t, i}^{p}=\vec{r}_{i j}^{t}\left(\vec{F}_{t, i j}+\vec{F}_{t, i j}^{d}\right) .
$$

Крутящий момент для сопротивления качению, согласно [26], имеет вид (19):

$$
\vec{T}_{r, i}^{p}=-\mu_{r}\left|\vec{r}_{i j}^{p}\right|\left|\vec{F}_{n, i j}\right| \frac{\vec{\omega}_{p q}}{\left|\vec{\omega}_{p q}\right|},
$$

где $\vec{r}_{i j}^{p}$ - вектор от центра масс частицы $p$ до точки контакта; $\mu_{r}-$ коэффициент трения вращения; $\omega_{p q}=\omega_{p}-\omega_{q}-$ угловая скорость частицы $p$ относительно частицы $q$. Крутящие моменты $\vec{T}_{t, e}^{p}, \vec{T}_{r, e}^{p}$ генерируются тангенциальной контактной силой и вращающимся трением соответственно.

Замыкания для силь сопротивления, действующей на частицу р по [17], представляется в виде (20):

$$
\vec{F}_{D}=A_{p} \overrightarrow{\hat{v}}
$$

где $\overrightarrow{\hat{v}}=\left(\vec{v}_{f}-\vec{v}_{p}\right)-$ скорость проскальзывания; $A_{p}-$ коэффициент обменных жидкостно-дисперсных эффектов, определяемый в виде (21):

$$
A_{p}=\frac{3}{4} c_{d} \frac{(1-\alpha) \rho_{f}|\overrightarrow{\hat{v}}|}{d_{p}} \alpha^{-1,65},
$$

где $d_{p}$ - диаметр частицы; $c_{d}-$ коэффициент сопротивления [28], выражающейся с учетом несферичности формы частиц (включая параметр $\varphi$ ) в виде (22):

$$
c_{d}=\frac{30}{\operatorname{Re}_{H B}}+\frac{67,289}{e^{5,03 \varphi}},
$$

где $\mathrm{Re}_{H B}$ - число Рейнольдса для частиц, отвечающее учету неньютоновского реологического фактора по модели Гершеля-Балкли (НВ). Последнее с отвечающими ему замыканиями (по данным [29]) имеет вид (23):

$$
\begin{gathered}
\operatorname{Re}_{H B}=\frac{\operatorname{Re}_{\mathrm{C} 3}}{1+\frac{7 \pi}{24} B i_{H B}}, B i_{H B}=\left(\frac{\tau_{0}}{k}\right)\left(\frac{d_{p}}{\mid \overrightarrow{\hat{v}}}\right), \\
\operatorname{Re}_{\mathrm{C} 3}=\frac{\rho_{f}|\overrightarrow{\hat{v}}|^{2-n} d_{p}^{n}}{k} .
\end{gathered}
$$

Здесь, как указано выше, $\tau_{0}$ - предельное напряжение изменений реологии среды; $k$ - параметр консистенции среды; $n$ - константа реологии степенного закона (С3).

Замыкания для крутящих моментов сопротивления можно определить, например, по [20] в виде (24):

$$
\vec{T}_{d t}^{p}=\frac{\rho_{p}}{2}\left(\frac{d_{p}}{2}\right)^{5} c_{d r}|\vec{\Omega}| \vec{\Omega},
$$

где $c_{d r}$ - коэффициент сопротивления вращения; $\vec{\Omega}-$ угловая скорость частицы относительно скорости жидкой фазы, имеющая вид (25), (26):

$$
\vec{\Omega}=\nabla \times \vec{v}_{f} / 2-\vec{\omega}_{p},
$$

$c_{d r}=\left\{\begin{array}{l}\frac{12,9}{\operatorname{Re}_{r}^{0,5}}+\frac{128,4}{\operatorname{Re}_{r}} \text { при } 32 \leq \operatorname{Re}_{r}=\frac{\rho d_{p}^{2}|\vec{\Omega}|}{\mu}<1000 ; \\ \frac{64 \pi}{\operatorname{Re}_{r}} \text { при } \operatorname{Re}_{r}<32 .\end{array}\right.$

Подъемная сила. Поперечные эффекты, как составляющие подъемной силы (Саффмена), $\vec{F}_{s}$, формируемые подъемной силой, а также ее составляющие от эффектов вращения (сила Магнуса), $\vec{F}_{m}$, которая расположена в нормальной плоскости к направлению движения относительной скорости между частицами дисперсной фазы и жидкостью, можно определить по $[20,25,30]$ в виде (27):

$$
\vec{F}_{s}=c_{l s} \frac{\rho_{f \pi}}{8} d_{p}^{3}\left[\overrightarrow{\hat{v}} \cdot \vec{\omega}_{f}\right], \quad \vec{\omega}_{f}=\nabla \cdot \vec{v}_{f}
$$

Здесь $\vec{\omega}_{f}$ - завихренность жидкости; $c_{l s}$ - коэффициент пропорциональности, по [20] имеет вид (28)-(30):

$$
\begin{gathered}
c_{l s}=\frac{4,1126}{\operatorname{Re}_{s}^{0,5} f\left(\operatorname{Re}_{H B}, \operatorname{Re}_{s}\right) ;} \\
f\left(\operatorname{Re}_{H B}, \operatorname{Re}_{s}\right)=\left\{\begin{array}{r}
\left(1-0,334 \beta^{0.5}\right) e^{-\mathrm{Re}_{H B}}+0,3314 \beta^{0,5} \\
\text { при } \operatorname{Re}_{H B} \leq 40 ; \\
0,0524\left(\beta \operatorname{Re}_{H B}\right)^{0,5} \text { при } \operatorname{Re}_{H B}>40 ;
\end{array}\right. \\
\beta=\frac{0,5 \operatorname{Re}_{s}}{\operatorname{Re}_{H B}}, \quad \beta \in(0,005 ; 0,4), \quad \operatorname{Re}_{s}=\frac{\rho_{f} d_{p}^{2}\left|\vec{\omega}_{f}\right|}{\mu} .
\end{gathered}
$$

Эффекты вращеени. Составляющую подъемной силы, обусловленную вращающимися эффектами (сила Магнуса) влияния на частицы дисперсной фазы $p$ по [20, 31], можно представить в виде (31), (32):

$$
\begin{gathered}
\vec{F}_{m}=\frac{\pi}{8} d_{p}^{2} \rho_{f} c_{l m}|\overrightarrow{\hat{v}}| \frac{[\vec{\Omega} \times \overrightarrow{\hat{v}}]}{|\vec{\Omega}|} ; \\
c_{l m}=0,45+\left(\frac{\operatorname{Re}_{r}}{\operatorname{Re}_{H B}}-0,45\right) e^{-0,5684 \mathrm{Re}_{r}^{0,4} \operatorname{Re}_{H B}^{0.3}} .
\end{gathered}
$$


Силу градиента статического давления жидкой фазы, воздействующей на частицу дисперсной фазы $p$ в локальной области ее движения, определяют в виде $F_{p}=-V_{p} \operatorname{grad} p$ при заданном объеме $V_{p}$ частицы.

Таким образом, решение внутренней задачи о гидродинамике и массопереносе в гетерогенной смеси в рамках ЭЛ/ЭЭ подходов, описываемой полной системой определяющих уравнений (2)-(32), строится численно с привлечением эффективных и экономичных методик, схем определения параметров задачи по следующему алгоритму. На первом этапе моделируется гидродинамика (используются оригинальные и коммерческие пакеты), затем ее результаты составляют опорные данные для расчета межфазного взаимодействия и деталей распределения частиц дисперсной фазы в рамках лагранжева подхода (определения поля скоростей частиц). Полученные новые значения положения частиц и их скоростей передаются в блок расчета гидродинамической части задачи, решение которой обновляет значение объемной доли частицы в расчетной ячейке. С учетом этих данных выполняются операции расчета гидродинамики на следующем временном шаге при условии достижения сходимости итерационного процесса в каждой точке пространства. Причем основную проблему расчета полных уравнений гидродинамики (Навье-Стокса), связанную с определением поля давления, решают с привлечением SIMPLE алгоритма С. Патанкара [18].

\section{Результаты моделирования и их анализ}

Оценки прогноза процессов при транспорте шламов

Согласно данным первого численного исследования гидродинамики при транспорте шламов по скважине с коаксиальным невращающимся ядром в условиях, характерных для бурения с забойными двигателями, а также с допущениями: раствор представлял собой среду со свойствами степенного закона, а не жидкости типа Гершеля - Балкли; одноразмерные частицы имеют сферическую форму, получено (рис. 2, [32]) вполне удовлетворительное соответствие опытам (погрешность меньше $10 \%$ ) значений интенсивности очистки при увеличении скорости течения смеси. Поставленные в [32] проблемы получили развитие у Н. Мишра в [33], где оценены возможности ЭЭ подхода в уяснении деталей процесса транспорта шлама и определены эффекты количественного влияния новых параметров (наряду с размером частиц и скоростью потока), таких как: вращение трубы, скорость бурения, угол наклонности на интенсивность течения и очистки. Заметим, что для моделирования турбулентности выбрана версия ке-модели [34]. Исследования имеют следующий недостаток. Так, в качестве жидкой фазы была выбрана капельная ньютоновская жидкость (вода). Анализировались процессы в ограниченном диапазоне параметров - скорости вращения ( 0,30 и 60 об/мин). Из рис. 2 следует, что численный прогноз при малых скоростях потока отличается ростом погрешности определения параметров задачи в сравнении с соответствующими опытными значениями. Валидация возможностей модели турбулентности на режимах с вращением внутренней стенки трубы не выполнялась. Все же подчеркнем, что результаты [33] важны для оценок возможностей эйлерова подхода, а также релаксации реофизических эффектов $[35,36]$ в задачах моделирования процессов в гетерогенных системах.

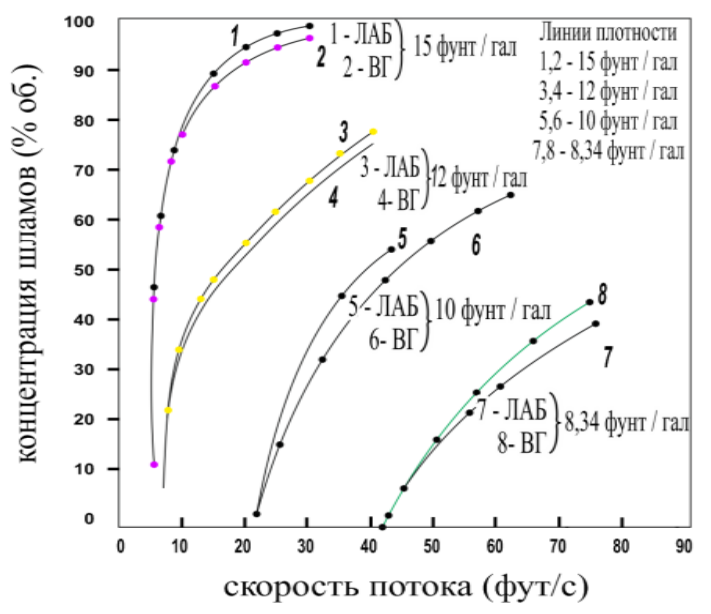

Pис. 2. Распределение интенсивности очистки в зависимости от различных значений скорости течения смеси (при переменной плотности смеси 8,34...15, фунт/гал). Здесь результаты методами численного вычислительной гидродинамики (ВГ) сравниваются с экспериментальными данными (ЛАБ) [32]

Fig. 2. Distribution of the cleaning intensity depending on different values of the mixture flow rate. Here, the results of numerical modeling of hydrodynamic processes $(B \Gamma)$ obtained are compared with experimental data (ЛАБ, [32])

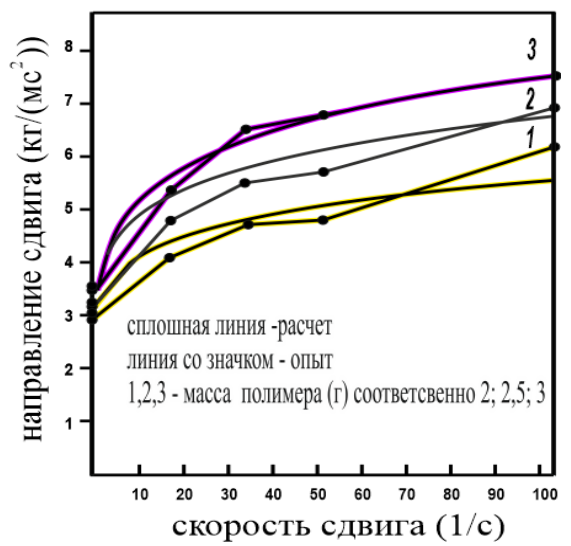

Puc. 3. Распределения напряжений в зависимости от скоростей деформачий при различных значениях концентрации массы полимера (по данным [37]): 1-3 (линии со значком) - результаты измерений; 1-3 (сплошные линии) - численное моделирование

Fig. 3. Stress distributions vs. a function of strain rates at different polymer mass concentrations according to [37]: 1-3 is the experiment (solid lines and symbols); lines 1-3 (without symbols) are simulation

В рамках ЭЭ подхода в [35] выполнены сравнения данных моделирования течений смесей с реологией степенного закона с экспериментами. Проанализиро- 
ваны режимы, осложненные эффектами: вращения трубы, переменности реофизических свойств, изменений концентрации частиц и их влияния на перепад давления. В [35] предполагается, что частицы составляют монодисперсную смесь, имеют сферическую форму, а геометрия межтрубного пространства отвечает условию $R_{\text {int }} / R_{\text {ext }}=0,7$. По [35] технологические условия бурения отвечают фактору «тонкого бурения». Это подразумевает, что перепад давления в кольцевом пространстве гораздо больше, чем в условиях «нормального» бурения. Так, в режиме нормального бурения 90 \% перепада давления происходит внутри буровых труб, а также в соплах долота, но только $10 \%$ в кольцевом пространстве. В этой области при тонком бурении перепад давления составляет порядка 60 \% перепада давления системы [36], что характерно для течений на горизонтальных участках скважин с диаметром меньше 6 дюймов. Отметим, что в [35] также выполнены оценки влияния изменений эксцентриситета $(e=0,0 \ldots 0,2)$ на эффективность очистки. Получено удовлетворительное соответствие данных моделирования течения с соответствующими экспериментами (погрешность меньше $10 \%$ ) при учете вращения внутренней трубы и его влияния на структуру ламинарного потока.

В [37] рассматривалось течение неньютоновской степенной жидкости в скважине с наклоном отдельных ее участков $30^{\circ}$. Причем первоначально анализировалось течение жидкой фазы как гомогенной среды (при отсутствии шламов) в межтрубном пространстве с неподвижной внутренней стенкой. Установлено, что изменения динамической структуры соответствуют данным экспериментов с погрешностью 1,9...8,4 \% при использовании в качестве рабочего тела растворов с разными значениями доли полимера (ксантановая камедь), причем точность результатов увеличивается с ростом концентрации полимера. Это объясняется тем, что при изменении концентрации полимера поведение жидкости больше отвечает неньютоновской реологии, характерной для модели степенного закона (рис. 3). В этой связи представляется важным исследовать процессы транспорта на основе жидкости со свойствами модели Гершеля-Балкли. На рис. 3 представлены (теория-опыт) распределения напряжений от скоростей деформаций при увеличении массы полимера $(2,0 \ldots 3,5$, г). Расчеты проведены при следующих данных: длина горизонтальной скважины - 30,48 м, диаметр внешней, внутренней труб и эксцентриситет соответственно - 0,203; 0,1143; 0,8 м; при неньютоновском течении в условиях ламинарного режима с массовым расходом - 15,13 кг/с, с параметрами реологии $-k=0,0254, n=0,72$; значениями частиц шлама: размер - 0,45 мм, плотность - 2650 кг $/ \mathrm{M}^{3}$, при механической скорости проходки (ROP) - 0,1267 кг/с. Видно, что в данном процессе результаты моделирования весьма чувствительны к изменению значений предела текучести.

В [38] выполнено численное исследование по установлению пространственной картины изменений траекторий частиц в потоке, осложненном вращением трубы в рамках ЭЛ подхода и реофизической модели
Гершеля-Балкли. Показано, что в коаксиальном пространстве траектории движения частиц имеют характер винтовой линии вдоль длины подвижной стенки трубы. В условиях вращения эксцентричной внутренней трубы закрученное движение вырождается по длине в прямоточное.

Учитывая опыт численного моделирования процессов транспорта шлама в рамках приближенных постановок и необходимость уяснения дополнительных эффектов, осложняющих течение, обусловленных турбулентностью (например, [32, 33]), в дальнейшем сформулированы проблемы включения в гидродинамические блоки рассматриваемых задач некоторых полуэмпирических моделей турбулентности. В связи с этим вполне разумными представляются исследования, в которых в рамках ЭЛ подхода сдвиговое течение смеси с неньютоновскими свойствами типа степенного закона выполняется по $\kappa \varepsilon / k w$ моделям [1, 2, 16, 18, 32, 33, 39]. При этом данные экспериментального анализа течений рабочей смеси (на трех различных растворах) позволили установить (например, $[32,33]$ ), что реофизические свойства такого сложного сдвигового потока отличаются от степенного закона на 3-8 \%. Это потребовало в дальнейших исследованиях обратиться к полимерным системам. Так, У. Мме и П. Скалле [40] провели моделирование внутреннего течения смеси с повышенной консистенцией и различными значениями сферичности частиц $(0,85 ; 0,9 ; 1,0)$. Установлено, что геометрия частиц сильно влияет на эффективность очистки, особенно при высоких скоростях потока. Кроме того, процесс очистки интенсифицируется в условиях повышенных значений сферичности частиц.

С целью выбора наиболее перспективной модели и ее замыканий представляется важным исследование [41], в котором в качестве тестирования результатов теории выбран ряд конфигураций процессов, для которых имеются аналитические решения и эксперименты.

Исследование [42] представляется первым, в котором учитывалась эксцентричность внутренней трубы $(e=0,9)$. Моделирование выполнено в рамках идей ЭЛ подхода, кє-модели [39], адаптированной к описанию турбулентности при сдвиговом потоке в смеси капельной ньютоновской жидкости с частицами сферической формы. Отметим, что реальные буровые трубы конструктивно не отличаются прямолинейностью, протяженностью и через интервал в 10 м содержат соединитель (большего поперечного размера), который оказывает влияние на течение в кольцевом пространстве (рис. 4). Параметрические расчеты с различной вязкостью жидкости, скоростями потока, вращения трубы показали: в реальных условиях полная очистка горизонтальных скважин от шламов невозможна без привлечения специального оборудования; большинство шлама накапливается в выше расположенной области перед первым соединителем.

Выяснению корректности моделей посвящены исследования [43, 44]. Так, в [44] моделирование течения смеси было выполнено в рамках ЭЭ подхода и привело к погрешностям прогноза локальных параметров с точностью до $30 \%$. 


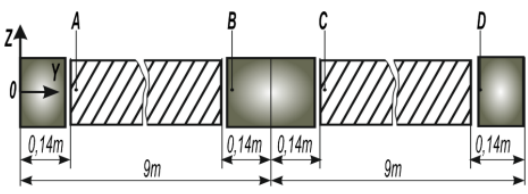

Рис. 4. Схема стандартной бурильной трубы с соединителями [42]

Fig. 4. Scheme of a standard drill pipe with joints [42]

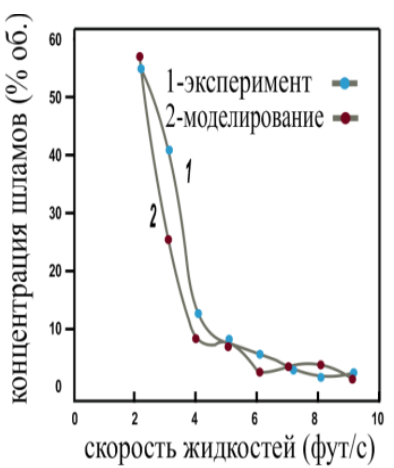

Pис. 5. Распределения концентрации шламов (\% об.) в зависимости от изменений среднемассовой скорости $($ фут/с) капельной жидкости (расчет 6 рамках лагранжева подхода - линия с темным иветом маркера) в сравнении с соответствующуими параметрами, измеренными опытным путем (данные - линия со светлым иветом маркеpa) [43]

Fig 5. Distributions of averaged cuttings concentration depending on changes in the bulk velocity of drip fluid (calculation within the Lagrangian approachdark marker's color) in comparison with the corresponding parameters measured experimentally (data - light marker's color) [43]

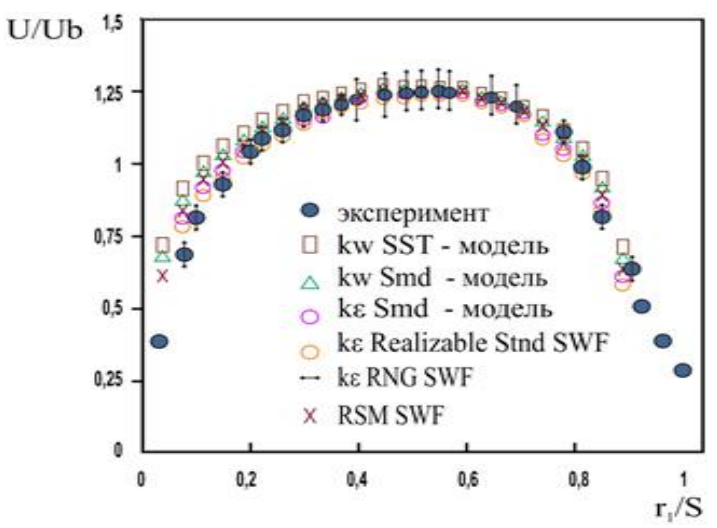

Pис. 6. Данные расчетов [45] распределений осевой компоненты вектора скорости, нормированной на среднемассовую скорость $\left(U / U_{b}\right)$ в радиальном направлении межтрубного пространства, описываемого безразмерным параметром $\left(r_{l} / S\right)$, полученных по моделям: $k w-S S T$ [46]; $k w$ [47-49]; $k \varepsilon$ [39]; $k \varepsilon-$ Realizable [2]; $k \varepsilon-R N G, R S M$ [2]

Fig. 6. Calculations [45] of distributions of the axial component of the velocity vector normalized by the average mass velocity $\left(U / U_{b}\right)$ in the radial direction of the annular space described by a dimensionless parameter $\left(r_{l} / S\right)$ obtained from the models: $k w$-SST [46]; $k w$ [47-49]; $k \varepsilon$ [39]; $k \varepsilon-$ Realizable [2]; $k \varepsilon-$ $R N G, R S M[2]$
В [43] акцент сделан на использовании ЭЛ подхода и тестировании результатов по экспериментам, выполненным на оборудовании в Средневосточном Университете (Стамбул, Турция). Обнаружено, что модель успешно предсказывает перепад давления, изменения полей концентрации шламов при высоких скоростях жидкостей. Однако результаты (соответствия теории и опыта) сильно расходятся при анализе режимов течений с низкими скоростями. Это связано с тем, что в [43] численно исследовался перенос шламов с неподвижного слоя к дисперсионному потоку с увеличением скорости жидкости. Анализ показывает, что данные расчетов нуждаются в совершенствовании модели транспорта смеси в рамках ЭЛ подхода, особенно при низких скоростях потока, при которых частицы сосредоточены в нижней части слоя из осаждаемых частиц в непосредственной окрестности стенки скважины, где их объёмная доля имеет значения больше $12 \%$. Эти сведения демонстрируют результаты, представленные на рис. 5, отвечающие значениям процесса: длина ствола и диаметр скважины соответственно - 0,6096; 0,073914 м; диаметр бурильной трубы и ее эксцентриситет соответственно - 0,04699; 0,623 м; буровой шлам составляют частицы гравия (диаметр - 0,0020066 м). Включаются условия: механическая скорость проходки - ROP $=0,00508-0,00677 \mathrm{~m} / \mathrm{c}$; расход воды по межтрубному пространству - 0,6096-2,7432 м/с; давление и температура в скважине соответственно -

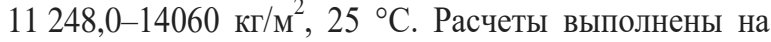
разностной сетке с числом конечных элементов 4107471. Данные расчетов показывают, что в таких условиях пространственные картины изменений полей скорости и состава при течении смеси с высокими скоростями (опыт) удовлетворительно предсказываются теорией.

Возможности расчета сложных течений в рамках отдельных моделей турбулентности RANS-метода представлены в [45]. Так, на рис. 6 приведены распределения осевой компоненты вектора скорости, нормированной на среднемассовую скорость, $U / U_{b}$, в радиальном направлении межтрубного пространства, описываемого безразмерным параметром, $r_{1} / S$. Здесь $r_{1}$ - радиальная координата, отсчитываемая в направлении от стенки внутренней трубы к внешней; $S$ - поперечный размер межтрубного пространства. Предполагается, что транспортируемая жидкость представляет собой неньютоновскую степенную вязкую среду, стенка внутренней трубы неподвижна и без эксцентриситета. Из рис. 6 видно, что в лучшем согласии с опытом находятся результаты расчета по SST $\kappa w$-модели [46]. Однако наш анализ сложных внутренних течений [2] указывает на преимущества моделей с уравнениями для псевдо завихренности $(w)$, интегрального масштаба $(L)$. Такие модели можно рекомендовать для прогноза очистки. Имеющиеся погрешности следует отнести на счет проблем моделирования распределений дисперсной фазы. Последняя рассчитывалась в рамках идей ЭЛ подхода, учета фактора столкновений частиц, описываемых моделью деформируемых сфер, аналогично [47]. Детальный анализ результатов исследования процессов гидроди- 
намики и массообмена показывает, что целесообразен учет моделью эффектов взаимовлияния процессов переноса между частицами несущей и дисперсной фаз. Кроме того, сравнения данных теории и опыта, отвечающего результатам [48], обнаруживают максимальную погрешность (порядка 5 \%). Заметим, что эксперименты проведены с одноразмерными частицами сферической формы, что приводит к заключению о необходимости совершенствования моделей динамики частиц смеси. Важным результатом этого исследования является оценка влияния на структуру течения вращений стенки внутренней трубы: вдоль своей собственной оси; вдоль оси внешней трубы (орбитальное вращение).

В [50] в рамках ЭЭ исследовалось течение пенного раствора с частицами дисперсной фазы. Учитывались эффекты от эксцентричности внутренней трубы $(e=0,78)$. Заметим, что данное значение более реально в практических процессах, нежели в [42], где геометрия трубы отвечала условию $e=0,9$. В [51] экспериментально проанализированы процессы в смеси, включающие одноразмерные частицы сферической формы (с диаметром 3 мм и плотностью 2610 кг/ м $^{3}$ ). Результаты показывают, что реология пены лучше описывается моделью степенного закона, нежели связью Гершеля-Балкли в режимах ламинарного течения, осложненного эффектами вращения стенки внутренней трубы. В таких режимах погрешность данных моделирования параметров поля течения составляет 8-10\%. Различия объясняются неточностью моделирования коэффициентов молярного переноса импульса и массы в смесях. Высокая вязкость пен и подвижность стенки внутренней трубы интенсифицируют конвективно-диффузионные механизмы процессов переноса массы и импульса в смеси. Включение этих деталей в математическую модель позволяет повысить точность расчета параметров локальной динамической и реофизической структур потока, особенно в режимах закрученного течения. Результаты таких исследований показывают, что использование пен с 90\%-м качеством (в практических режимах бурения с пеной, отвечающих закрутке трубы со скоростью 120 об/мин, течении со скоростями 2 фут/с) повышает очистку скважин от шламов до 80 \%. Важным следует признать замечание авторов о закономерностях влияния изменений реофизических свойств на несущую способность пенных растворов. Так, в отличие от полимерных растворов, которые лучше соответствуют модели Гершеля-Балкли, пены целесообразно описывать моделями: Бингама [52]; степенным законом [53]; Гершеля-Балкли [54]. В этом отношении данные [51] расчетов по ЭЭ подходу течений пен различного качества показывают, что пены с качеством меньше $80 \%$ лучше реализуются моделью Гершель-Балкли, тогда как модель степенного закона характерна для пен с качеством выше 80 \%.

В $[55,56]$ численно проанализированы эффекты влияния переменности параметров: отношения диаметров труб, скорости потока, вращения стенки внутренней трубы и реологии жидкости, на интенсивность образования массы шламов, а также величины изме- нения поля давления в горизонтальных скважинах с эксцентричным размещением внутренней трубы. Математическое моделирование транспорта шламов выполнено на базе ЭЭ подхода в межтрубном пространстве с эксцентриситетом $e=0,623$ и отношением диаметров труб 0,64/0,9. Такие значения соответствуют реальным условиям при бурении горизонтальных участков, а также ситуациям, когда внутренняя труба почти соприкасается с нижней поверхностью внешней трубы и лишь элементы соединителей определяют ее отклонение от опорной поверхности. В $[55,56]$ оценено влияние заданий реальных значений геометрической конфигурации элементов оборудования, составляющих блок определения краевых условий для численного интегрирования дифференциальных уравнений математической модели. Отдельные результаты приведены на рис. 7, $a-d$, где отображена конфигурация межтрубного пространства с эксцентричным ядром (e=0,623) при различных значениях отношения диаметров $(0,64$ (a); 0,7 (b); 0,8 (c); 0,9 (d)) c картиной разностных сеток и особенностей размещения контрольных объемов в пристеночных зонах коаксиального пространства. Такие сведения о сетках полезны для уяснения проблем формулировки краевых условий, деталей построения устойчивого численного алгоритма расчета сложного сдвигового низкорейнольдсового течения (например, $[1,2])$ неньютоновской среды со степенным законом реологии, а также выбора оптимальной версии $k \varepsilon$-модели [57] в анализе турбулентности.

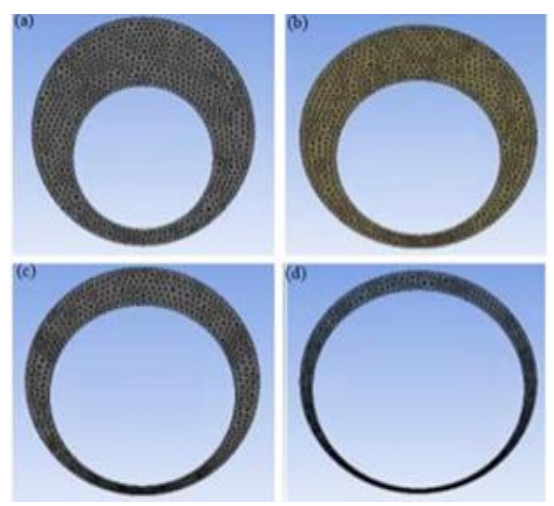

Рис. 7. Конфигурачия межтрубного пространства с эксиентричным ядром $(e=0,623)$ при отношении диаметров: 0,64 (a); b) 0,7 (b); 0,8 (c); 0,9 (d), nо даннымм [55]

Fig. 7. Configuration of the annular space with an eccentric core $(e=0,623)$ for different values of the diameter ratio: $0,64(a) ; 0,7(b) ; 0,8(c) ; 0,9(d)$, according to [55]

В [58] выполнено сравнение результатов моделирования (рабочее тело - вода) с данными опытов других авторов. Обнаружено, что соответствие теории и опытов по перепаду давления имеет погрешность меныше (2,18-4,4 \%), а среднемассовых концентраций шламов меньше $(6,4-11,82 \%)$. Установлено, что вращение трубы интенсифицирует процесс транспорта шламов только при низких скоростях потока. Отдельные результаты численного моделирования, удо- 
влетворительно описывающие реальный процесс транспорта шламов, приведены на рис. 8.

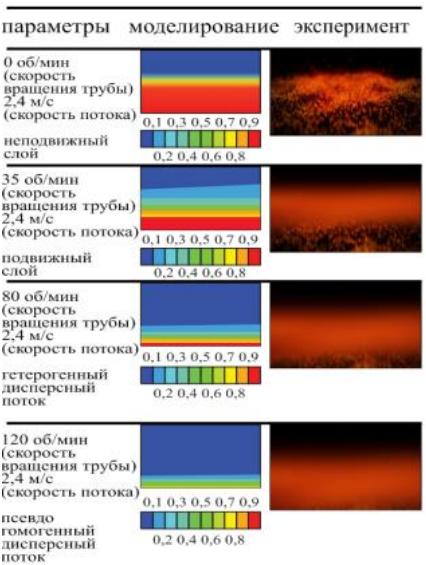

Pис. 8. Изменение режима потока слоя шламов при различных значениях вращения трубы. Шкала под результатами моделирования показывает объёмную долю шлламов [58]

Fig. 8. Distribution of the cutting flow mode at different pipe rotation values. The scale under the simulation results shows the volume fraction of cuttings [58]

Таким образом, представленный анализ результатов численного и экспериментального исследований процессов очистки в режимах сложного течения указывает на большой спектр условий, формирующих погрешности расчетов локальных и интегральных параметров задачи [4-15, 17, 30-38, 40-45, 47, 48, 50-56, $58,59]$. И это неудивительно, т. к. в своем анализе многие авторы обращаются к феноменологическому методу.

Моделирование транспорта смесей в проблемных технологиях бурения

С этих позиций интересны сведения [60], где обращено внимание на перспективное направление, обусловленное бурением с колтюбингом (с гибкими трубами), хотя главным препятствием на пути его широкого применения остаются несовершенство технологий и проблемы очистки скважин. Последние обостряются из-за невозможности обеспечить вращение трубы при образовании слоя шламов и заставляют искать решение в направлении выяснения условий для установления минимальной скорости потока, при которой обеспечивается вынос шламов из скважины или предотвращение их образования. С этой целью в [60] обращаются к ЭЭ подходу и низкорейнольдсовой $k w$ SST-модели турбулентности [46]. Расчеты выполнены для трех типов жидкостей: воды, жидкости со степенным законом и связью Гершеля-Балкли. Следует отметить, что в условиях традиционного бурения (без вращения трубы) турбулентностью можно пренебречь. Тогда как при колтюбинге, вследствие узости кольцевого межтрубного пространства, турбулентность и ее механизмы при транспорте шламов имеют существенное значение и требуются обоснованные заключения о точности их расчета. Так, возможные переходы от ламинарного к турбулентному режиму в потоке оценивались экспериментально (таблица), причем следует учесть, что для всех жидкостей представленное число Рейнольдса рассчитано по свойствам ньютоновской среды. Результаты показывают, что численное моделирование с удовлетворительной точностью способно прогнозировать режимы течений с минимальной скоростью, необходимой для очистки элементов оборудования от шламов.

Таблица. Распределения чисел Рейнольдса и его связи с характерной скоростью потока в режимах: перехода с ламинарного к переходному; от переходного к турбулентному, для трёх различных по реологии жидкостей [60]

Table. Distribution of Reynolds numbers and its relation to the characteristic flow velocity in transition from laminar to transient and from transient to turbulent for three fluids of different rheology [60]

\begin{tabular}{|l|c|c|c|c|}
\hline \multirow{2}{*}{\begin{tabular}{|} 
Жидкость \\
Fluid
\end{tabular}} & \multicolumn{3}{|c|}{ Режимы течений потока/Flow regimes } \\
\cline { 2 - 5 } & \multicolumn{2}{|c|}{$\begin{array}{c}\text { от ламинарного } \\
\text { кереходному } \\
\text { from laminar } \\
\text { to transition }\end{array}$} & \multicolumn{2}{|c|}{$\begin{array}{c}\text { от переходного } \\
\text { к турбулентному } \\
\text { from transition } \\
\text { to turbulent }\end{array}$} \\
\cline { 2 - 5 } & $\begin{array}{c}\text { число } \\
\text { Рейнольдса } \\
\text { Reynolds } \\
\text { number }\end{array}$ & $\begin{array}{c}\text { скорость } \\
\text { (см/c) } \\
\text { velocity } \\
\text { (cm/s) }\end{array}$ & $\begin{array}{c}\text { число } \\
\text { Рейнольдса } \\
\text { Reynolds } \\
\text { number }\end{array}$ & $\begin{array}{c}\text { скорость } \\
\text { (см/c) } \\
\text { velocity } \\
\text { (cm/s) }\end{array}$ \\
\hline $\begin{array}{l}\text { Ньютоновская } \\
\text { Nеwtonian }\end{array}$ & 2099 & 7 & 3000 & 9 \\
\hline $\begin{array}{l}\text { Степенная } \\
\text { Роwеr law }\end{array}$ & 2411 & 49 & 3311 & 62 \\
\hline $\begin{array}{l}\text { Гершеля-Балкли } \\
\text { Неrschel-Вulkley }\end{array}$ & 2589 & 86 & 3489 & 105 \\
\hline
\end{tabular}

Анализ эффектов, сопровождающих течение в трехфазной смеси (жидкий раствор, твердые частицы и газ), осложненное тепломассопереносом, впервые выполнен в [61] для кольцевого пространства скважины. Подобная ситуация характерна для бурения скважин в режиме депрессии, который применяется при бурении обеднённых пластов. Численное моделирование внутри- и межфазных процессов переноса импульса, тепла и массы проведено в рамках ЭЭ подхода (для капельной и газообразной фаз), ЭЛ подходом - явлений в твердой фазе. Турбулентность оценивалась с помощью $k \varepsilon$-модели [39]. Допускается, что частицы имеют сферическию недеформируемую форму и предрасположены к столкновениям друг с другом. Решение задачи построено по алгоритму с первоначальным расчетом процессов в газожидкостной системе, позволяющей определить силы влияния со стороны капельной смеси на частицы (сопротивления, подъёмная сила, сила градиента давления). В [62] при исследовании процессов, включающих частицы в форме сферических керамиче-

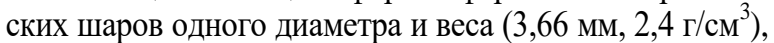
установлено, что погрешность (соответствие локальных и интегральных данных теории и опыта) при низких скоростях потока возникла из-за некорректности выбора коэффициентов сопротивления трения. Вместе с этим стоит допустить, что аналогично [43] при низких скоростях потока ЭЛ подход не вполне приемлем, так как погрешность определения концентрации частиц превышает $12 \%$. 
Общий анализ результатов очистки позволяет отметить [47, 48, 50-56, 58-62], что процессы транспорта в реофизически сложных смесях во внутренних системах с эксцентричным ядром вполне успешно (погрешность до 4 \% изменения интегральных параметров) описываются в рамках замыканий, используемых в программных пакетах по гидродинамике. Физически содержательные процессы в многофазном потоке исследованы в [63] на базе специального оборудования типа Позитронной Эмиссионной Томографии. Опираясь на эти результаты, в [64] выполнены исследования течений многофазной среды в рамках ЭЛ подхода, $\kappa w$-модели турбулентности [49] со сферическими частицами и степенной жидкости. Обнаружено, что изменения локальных и интегральных параметров на различных этапах развивающего течения смеси в сравнении с соответствующими опытами могут быть значительными (с погрешностью от 5 до 40 \%).

В [65] на основе модели Сямлал - Обраян [66] предпринята попытка установить причину значительной погрешности численного моделирования нелинейных процессов в смеси, анализируя эффекты от переменности формы частиц. В качестве жидкой фазы использовались среды с реологией степенного закона и Гершеля-Балкли. Сравнение результатов моделирования течения смеси ньютоновской жидкости с частицами разных размеров с экспериментами [67] показало точность прогноза процесса перепада давления до $8,1 \%$, концентраций частиц - до 10,3 \%. Все же это еще недостаточно для выдачи рекомендаций по корректному управлению процессами.

$\mathrm{B}$ [23] для уяснения деталей влияния формы частиц использовали ПК (STAR-CCM), ЭЛ подход и четыре формы частиц (рис. 9, данные [22]). В исследованиях предполагается, что для каждой частицы, состоящей из совокупности сфер, можно вычислить отдельные силы межфазного взаимодействия с замыканиями (7)-(32). Результаты расчета сравнивались с опытами [68]. Обнаружено, что форма частиц очень сильно влияет на результаты. Имеется ряд публикаций, в которых в качестве бурового раствора используются жидкости, включающие смесь воды и различных добавок, например [69, 70].
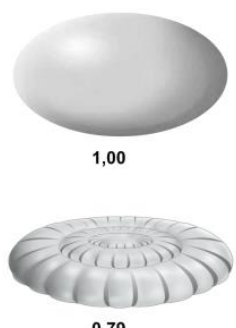

0,79

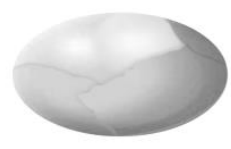

0,98

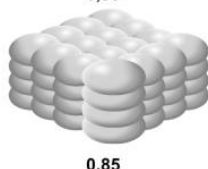

0,85
Pис. 9. Разнообразные геометрические формы частии, используемых в численном моделировании и построенных из меньших сфер. Число под изображением - коэффиичент сферичности (по данHolм [22, 23])

Fig. 9. Various geometric shapes of particles used in numerical modeling and constructed from smaller spheres. The number under the image is the sphericity coefficient (according to [22, 23])

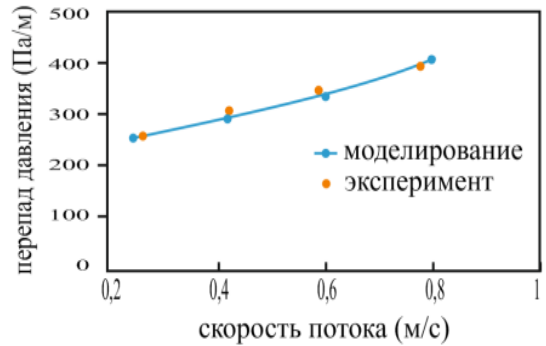

Pис. 10. Распределение перепада давления от скорости потока для условий, сопровождающих бурение и включаюших неньютоновские среды в качестве бурового раствора. Здесь: линия с символом расчет ламинарного течения однофазной жидкости типа Гершеля-Балкли в эксиентричном кольцеевом пространстве; символь - эксперимент [71, 72])

Fig. 10. Distribution of the pressure drop vs flow rate for real drilling conditions using a non-Newtonian drilling fluid. Here: the calculation (line with symbol) corresponds to the laminar flow of a singlephase Herschel-Bulkley liquid in an eccentric ring space; symbols correspond to the experiment [71, 72]

Наряду с этим существуют растворы с типом нефти или газойля, не содержащие твёрдые частицы и используемые при бурении продуктивных слоёв. В [71] впервые выполнено исследование определения эффективности их функционирования при транспорте шламов. При моделировании использован ЭЭ подход, реологическая модель Гершеля-Балкли. Результаты распределений перепада давления, вызывающего транспортировку общей массы бурового раствора со свойствами подобной неньютоновской жидкости по межтрубному пространству скважины в зависимости от его среднемассовой скорости, иллюстрируют данные рис. 10, где моделирование (линия) отвечает экспериментальным условиям течения (символы, данные $[71,72])$. Высокая степень соответствия данных теории и опыта свидетельствует об адекватности модельного описания и прогноза процессов переноса импульса смеси, характера изменений поля скорости в кольцевой зоне.

Более полная картина процессов течения шламов в смеси с неньютоновской жидкостью типа ГершельБалкли в скважине с эксцентричным ядром рассматривалась в [73, 74]. Моделирование гидродинамики турбулентного течения выполнено в рамках ЭЭ подхода, включающего обращение к моделям вихревой вязкости $(k \varepsilon, k w[39,46,49])$, полного тензора напряжения Рейнольдса $[1,2,57]$ - для учета анизотропных эффектов. При описании движения частиц твердой фазы предполагалась их одноразмерность $(0,25$ дюймов с плотностью $\left.2650 \mathrm{\kappa} / \mathrm{m}^{3}\right)$ и сферическая форма. Эффекты межфазного взаимодействия описываются в рамках положений моделей Гидаспау [75], а также модели Сямлал [66]. Некоторые данные распределений концентраций частиц смеси в зависимости от среднемассовой скорости течения смеси в межтрубном пространстве приведены на рис. 11, где имеются сравнения расчетов с данными экспериментов, выполненных в [76, 77]. Из рисунка видно, что модели- 
рование диффузии вещества смеси вполне успешно ввиду полного учета нелинейных конвективнодиффузионных механизмов изменений полей скоростей фаз, их межфазных эффектов. Это позволяет судить о надежности ЭЭ подхода в прогнозах процессов в данной гидродинамической конфигурации.

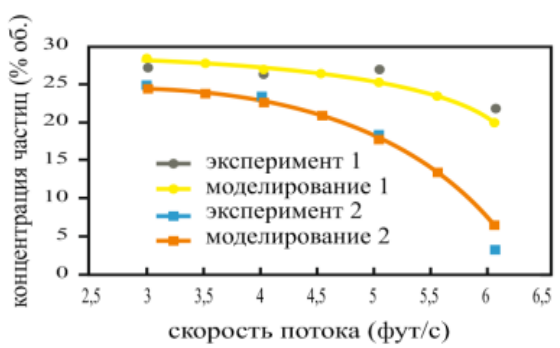

Рис. 11. Распределения концентраций частии в зависимости от скорости потока: 1 - с качеством пены $80 \%$; 2 - с качеством пены $90 \%$ при использовании частии размером 3 мм и плотностью 2,61 кг/ $\mathrm{cм}^{3}$; длинна труб 73 футов, диаметр 5,6 u 3,5 дюймов; без вращения внутренней трубы. Результаты расчета сравнили с результатами экспериментов [76], сведения по данным [73]

Fig. 11. Distributions of particle concentrations vs average flow velocity: 1 -with foam quality of $80 \% ; 2$ - with foam quality of $90 \%$ using particles of $3 \mathrm{~mm}$ in size and density of 2,61 kg/ $\mathrm{cm}^{3}$; length of pipes is $73 \mathrm{feet}$ and diameters are 5,6 and 3,5 inches; without rotation of the inner pipe. The calculation results were compared with the experimental ones [76], the data corresponds to [73]

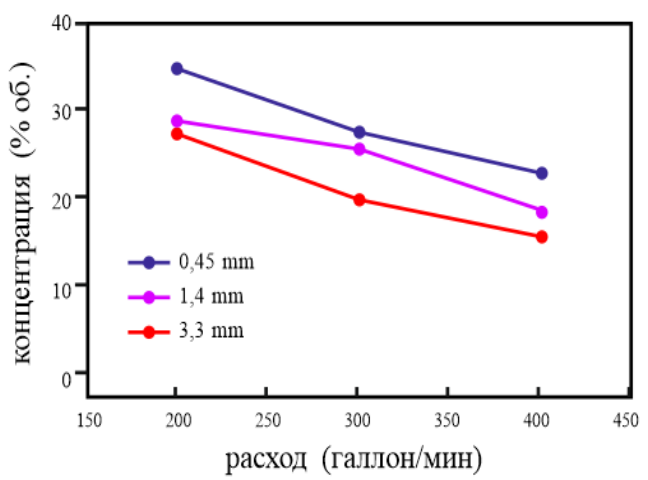

Рис. 12. Распределения объемной концентрации шлама в зависимости от изменений расхода, данные [74]

Fig. 12. Cuttings volumetric concentration vs. water flow rate for three different cuttings. Data from [74]

Отдельные авторские результаты численного исследования транспорта шлама (в условиях опыта [74]) при сложном сдвиговом течении, описываемом $k \varepsilon$ моделью RANS-метода и ЭЭ подходом приведены на рис. 13-17, где проиллюстрирован характер изменения локальной структуры осредненного (рис. 13) и пульсационного течения смеси (рис. 14, 16, 17), частиц твердой фазы (рис. 15), а также ее отдельных интегральных параметров (рис. 12, данные [74]). В частности, расчеты в коаксиальной эксцентричной области скважины распределений объемной концентрации шлама в зависимости от изменений расхода (рис. 12), осевой скорости (рис. 13), турбулентной вязкости (рис. 14), кинетической энергии турбулентности (рис. 16), степени турбулентности (рис. 17) для дистальной зоны $(\mathrm{x} / \mathrm{D}=150)$ и концентрации частиц твердой фазы (рис. 15) в продольном сечении скважины $x / D=73,8$ в турбулентном режиме течения капельной ньютоновской смеси с частицами различных размеров представлены при следующих условиях. Анализировался транспорт в воде частиц песка размера и плотности соответственно, мм: 3,$3 ; 1,4 ; 0,45$, $\rho=1538$ кг $/ \mathrm{M}^{3}$; при $\mathrm{Re}=5,410^{4}, 7,610^{4}, 110^{5}$; в межтрубном пространстве горизонтального участка: эксцентриситет - 0,8; внешний (D)/внутренний (d) диаметры соответственно, м - 0,2032/0,1143; длина скважины - 30,48 м. Результаты показывают, что замыкания математической модели и вычислительная технология построения решения задачи весьма успешны в прогнозе интегральных процессов. Об этом свидетельствуют сравнения с опытными данными [74] эволюции прямоточного течения смеси, изменений концентрации частиц. Так, аналогично данным рис. 12, расчетами обнаружена тенденция к снижению концентрации шлама в представленном диапазоне изменений расхода с отличием в 6-7 \% между двумя близкими распределениями концентраций. Кроме того, установлено, что в данном режиме транспорт частиц в смеси сопровождается изменениями параметров пульсационной структуры, точность которых не удается оценить из-за отсутствия соответствующего опытного материала [74]. Отметим, что последнее затрудняет непосредственное сравнение расчета объемной концентрации с опытом [74]. Данные моделирования указывают, что даже в условиях прямоточного течения (RPM=0) в межтрубном пространстве структура турбулентного течения отличается существенной неоднородностью в узких зонах поперечного сечения (рис. $13,14,16,17)$, что соответствует [74]. Также установлено, что подавление частицами инерционных и диффузионных эффектов (рис. 15) обостряет механизмы обратного перехода в турбулентном течении и способствует ламинаризации $[1-3,18]$.

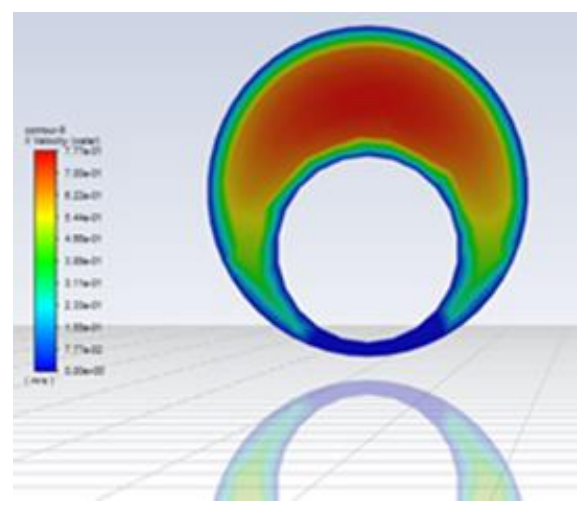

Pис. 13. Радиальное распределения осевой компоненты вектора скорости в выходном сечении $(x / D=150)$ коаксиальной области при $R e=1,1 \cdot 10^{5}$

Fig. 13. Radial distribution of axial component of velocity vector in output section $(x / D=150)$ of coaxial region at $\operatorname{Re}=1,1 \cdot 10^{5}$ 


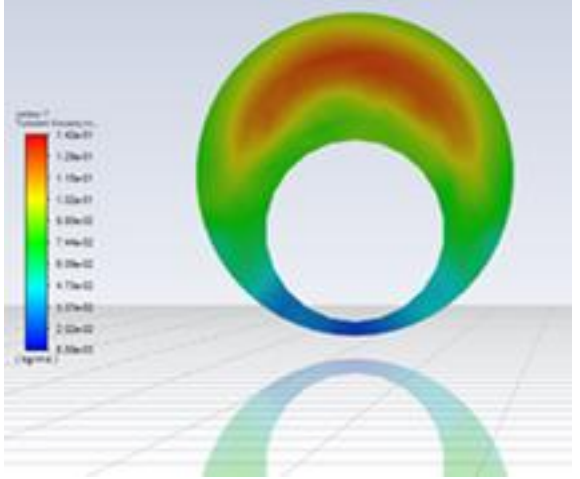

Рис. 14. Поле молярной вязкости в выходном сечении nри $\operatorname{Re}=7,6 \cdot 10^{4}$

Fig. 14. Molar viscosity in output section at $R e=7,6 \cdot 10^{4}$

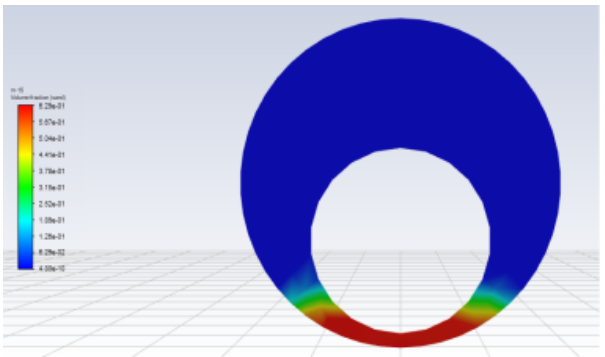

Рис. 15. Радиальное распределение кониентрации частии песка при $x / D=150, \operatorname{Re}=5,4 \cdot 10^{4}$

Fig. 15. Radial distribution of sand particles concentration at $x / D=150, \operatorname{Re}=5,4 \cdot 10^{4}$

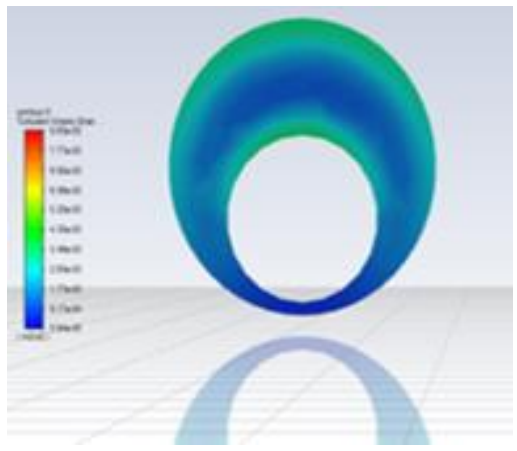

Рис. 16. Поле кинетической энергии турбулентности в выходном сечении при $R e=5,4 \cdot 10^{4}$

Fig. 16. Turbulence kinetic energy in output section at $\operatorname{Re}=5,4 \cdot 10^{4}$

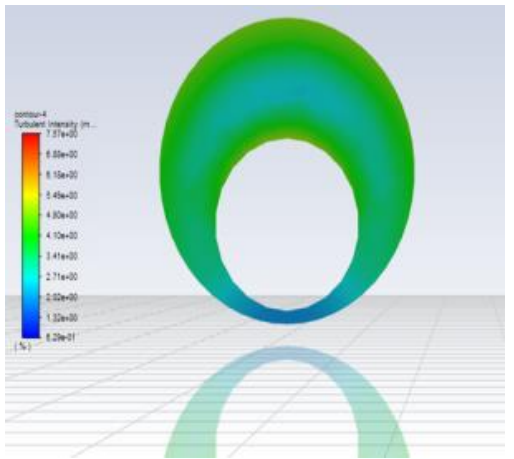

Рис. 17. Поле интенсивности турбулентности в выходном сечении при $R e=1,1 \cdot 10^{5}$

Fig. 17. Turbulent intensity in output section at $R e=1,1 \cdot 10^{5}$
В [78] использована близкая к [73] по физическому содержанию модель транспорта смеси капельной жидкости типа Гершеля-Балкли и одноразмерных сферических частиц в коаксиальной области с эксцентричным вращающимся ядром при ламинарных и турбулентных режимах течения дисперсных сред. Причем для расчета молярных свойств течения ньютоновской смеси привлекалась однофазная двухпараметрическая $k w$-SST модель [46], а для неньютоновских дисперсных систем - система феноменологических модельных замыканий вида (33)-(35). Так, для уравнения движения смеси имеем тензор вязких напряжений по [79, 80]:

$$
\begin{gathered}
\overline{\bar{T}}=2 \mu\left[\overline{\overline{\dot{S}}}-\frac{1}{3}(\nabla \cdot \vec{v}) \overline{\bar{I}}\right], \\
\mu=\mu_{f} \exp \left\{\frac{2,5}{\beta}\left[\frac{1}{\left(1-\alpha_{s}\right)^{1,5}}-1\right]\right\}+\mu_{f r} ; \\
\mu_{f}=\frac{k_{v} \dot{\gamma}^{n}+\tau_{0}\left(1-e^{-m \frac{\dot{\gamma}}{\dot{\Gamma}}}\right)}{\dot{\gamma}} \\
\dot{\gamma}^{2}=2 S_{i j}^{d} S_{i j}^{d}+\frac{\rho \varepsilon}{\mu} .
\end{gathered}
$$

Здесь $\rho$ - плотность смеси; $\mu$ - вязкость смеси; $\varepsilon-$ скорость диссипации кинетической энергии турбулентности; $\mu_{f}-$ вязкость жидкости; $\mu_{f r}-$ вязкость, обусловленная взаимодействием частиц; $\dot{\gamma}-$ скорость деформаций в смеси; $\beta, n, m, \dot{\Gamma}-$ постоянные реологической модели неньютоновской среды; $k_{v}$ - параметр консистенции смеси; $\tau_{0}$ - предел текучести.

Расчеты выполнялись при следующих параметрах течения смеси: критерий Рейнольдса, $\mathrm{Re}=\frac{\rho_{f} U^{2-n} D_{0}^{n}}{\kappa}=$ $=740 . .8000$; критерий Бингама, Вi $=\frac{\tau_{0}}{\kappa\left(\frac{U}{D_{0}}\right)^{n}}=15 \ldots 5,5$; $\rho_{f}=2400 \kappa \Gamma / \mathbf{M}^{3}, \tau_{0}=7,34$ Па, $n=0,6435 ; k_{v}=0,0747$ Па $\mathrm{c}^{\mathrm{n}}$; внешний и внутренний диаметры соответственно, $D_{o}=0,254 \mathrm{~m} ; D_{i}=0,127 \mathrm{~m} ; U=0,957 \mathrm{~m} /$; диаметр частиц, $d_{p}=3 \mathrm{Mм}$.

Численное моделирование определяющих уравнений законов сохранения выполнено в рамках ЭЭ метода на мелких разностных сетках, что позволило добиться точности расчета локальных параметров. Причем установлено [78], что вследствие роста параметров текучести среды (при изменении вязкости и скорости вращения бурильной трубы) наблюдается уменьшение концентрации частиц шлама в поперечном сечении канала. Исследование показало, что при транспорте шлама наблюдаются два режима, формируемых: первичной циркуляцией (из-за вращения буровой колонны), а также вторичной, где доминируют вторичные вихревые структуры. Физические особенности первого режима определяются наличием небольшой концентрации частиц в поперечном сечение канала и сравнительно небольшим перепадом давления. Такие значения являются предпочтительными для бурения и могут быть достигнуты при небольших 
эксцентриситетах и высоких скоростях вращения. Второй режим поддерживается малыми скоростями вращения, малой вязкостью жидкости и высокими концентрациями частиц дисперсной фазы. Отмечено, что поток в эксцентричном кольцевом пространстве всегда генерирует вторичную циркуляцию, которая включает эффекты перехода вихревой природы и оказывает значительное влияние на падение давления, динамику и концентрацию частиц в локальных областях течения. Общий анализ результатов процессов течений смеси по [73, 78] указывает на корректность прогноза транспорта частиц в сложном закрученном течении смеси из-за вращения внутренней стенки трубы вдоль своей оси, а также влияния ее эксцентриситета и орбитального вращения буровой трубы в скважине.

Деталям исследования гидродинамики турбулентного гетерогенного потока, осложненного орбитальным вращением внутреннего ядра/трубы, посвящена работа Б. Панга и соавторами [81]. Моделирование турбулентного течения реофизической смеси с внутри- и межфазными процессами переноса импульса и массы выполнено в рамках идей ЭЭ подхода, модели межфазного взаимодействия Гидаспау со сферическими, одноразмерными частицами (подобно [73]), $k w$ модели [49] и степенного реологического закона. Верификация результатов моделирования оценивалась по данным измерений локальных параметров течения выполненных в [82]. Соответствие изменений расчетных интегральных параметров экспериментальному режиму (с параметрами: раствор 0,4 КМК и 0,5 \% бентонайт, расход жидкости 1 лит/мин, длина труб 1,8 м, диаметр труб 44 и 30 мм, диаметр частиц 0,1 мм и плотность 2,55 кг/ $\left.\mathrm{cm}^{3}\right)$ с погрешностью до $15 \%$ говорит об удовлетворительном качественном описании эффектов переноса в смеси. Последнее можно объяснить тем, что моделирование выполнено в среде со свойствами модели Гершеля-Балкли. Общий анализ результатов $[78,81]$ также подтверждает факт влияния орбитального движения трубы на очистку скважины. Причем тем значительнее, чем интенсивнее её вращение, в частности, вдоль собственной оси.

С учетом сформулированных выше модельных допущений (ЭЭ подход, сферические частицы, неньютоновская степенная жидкость, $k w$-SST-модель турбулентности, реофизическая модель Гидаспау) в [83] проведен анализ процессов транспорта во внутренних системах (трубы с соединителями). Согласно этим данным видно, что в большинстве исследований рассматриваемых процессов авторы обращаются к моделям межфазного взаимодействия, представленным Гидаспау, либо Сямла-Обрайн. Результаты расчетов гидродинамики и массопереносв в трубах показывают, что центральное место в проблеме повышения точности прогноза параметров течения и транспорта частиц по межтрубному пространству скважины занимают механизмы, ответственные за обменные эффекты, обусловленные орбитальным вращением поверхности стенки внутренней трубы. Заметим, что два ярких свойства орбитально вращающихся пото- ков выделяют такое движение в класс особых режимов течений жидкостей и газов: первое обусловлено созданием поля центробежных сил, подавляющих действие силы тяжести, и одновременно генерацией механизмов, интенсифицирующих вторичные течения; второе ответственно за изменение характера пристеночного течения и механизмов в периферийных коаксиальных зонах. Данные свойства целенаправленно используются при проектировании механизмов, препятствующих осаждению частиц в жидкости (буровом растворе). Экспериментальное исследование особенностей течения при орбитальном вращении вязкого потока на полномасштабных установках затруднено из-за необходимости внесения новых технологических и конструкторских решений по эффективному вращению внутренней трубы. В настоящее время такие эффекты составляют предмет теоретического анализа, а экспериментально моделируются только процессы с коаксиальной закруткой. Эти данные привлекаются для тестирования процессов очистки скважин.

Результаты опубликованных работ в последние годы (например, [84-89]) показывают существенный прогресс компьютерного моделирования процессов транспорта шламов при обыкновенном бурении, а также адаптации популярных гидродинамических подходов и моделей к малоизученным проблемам. Например, Б. Панг и соавторы [84] изучили процесс очистки скважин при бурении, осложненном пульсационным режимом движения раствора. Б. Шаю и соавторы [85] моделировали подобный процесс при бурении метаноугольных пластов с применением двойной циркуляции, а А. Гайлани и соавторы [86] исследовали этот процесс с привлечением современных технологий в рамках смесей с наночастицами [86]. Bсе современные исследования нуждаются в выяснении вопроса достоверности используемых численных методик, особенно при выдаче рекомендаций в практику. В этом смысле, например, М. Эскудиер и соавторы [87] обращают внимание на необходимость детального моделирования турбулентной неоднородности и анизотропии во внутреннем потоке при транспорте реологически сложной среды. М. Хук и соавторы [88] проанализировали чувствительность очистки шламов к разным параметрам (особенно к размеру частиц). Ф. Жанг и соавторы [89] провели большую работу по апробации новых моделей вычислительной гидродинамики в прогнозе радиальных процессов в скважинах и получили более точные результаты изменений локальной структуры потока. Все последние результаты демонстрируют, что поиск более точных, эффективных и экономичных маршевых схем, методов моделирования и расчета процессов очистки скважин представляет интенсивно развивающуюся и весьма актуальную тему инженерных гидродинамических исследований.

Таким образом, детальный анализ указанных проблем по [1-3, 60-89] позволяет утверждать, что в настоящее время имеется большой дефицит экспериментальных данных, необходимых для тестирования изменений локальной структуры потока, валидации 
разрабатываемых математических моделей и верификации их результатов. Вместе с этим очевиден прогресс исследований сложных течений смесей в межтрубном пространстве (в конфигурациях, характерных для скважин) в рамках ЭЭ и ЭЛ подходов, позволяющих вполне корректно прогнозировать нетривиальные эффекты при транспорте шлама в достаточно широких гидродинамических условиях, особенно при наложении крутки на прямоточное течение смеси.

\section{Выводы}

Анализ результатов численного моделирования процессов очистки горизонтальных скважин позволяет сделать следующие выводы:

1. Прогноз процессов в жидкой фазе считается вполне успешным для большинства локальных параметров задачи (полей компонентов вектора скорости, концентрации и т. д.) и интегральных величин (перепада давления, сопротивления и т. д.), характеризующих течения в смесях в широком диапазоне изменений их реофизических свойств, геометрических и гидродинамических конфигураций, изменений межтрубного пространства, размеров труб, их соединений, особенностей размещения бурового оборудования. Хорошее соответствие результатов моделирования транспорта шламов экспериментам дают результаты исследований $[22,23,33,71-73$, 77, 80-83], отвечающих формированию транспорта частиц шлама в условиях вязко-инерционного течения. Большей частью это характерно для режимов транспорта при параметрах: использования ньютоновской и неньютоновской жидкостей Гершеля-Бакли, со степенным законом, пен и их смесей $\mathrm{c}$ массой полимера 2,0..3,5 г; при $\mathrm{Re}=740 \ldots 110000, \mathrm{Bi}=3 \ldots 20, e=0,0 \ldots 0,9 ;$ в геометриях с изменениями внешнего/внутреннего диаметра труб $\sim(12,25 \ldots 8,5) /(5,5 \ldots 5,0)$; длины скважины - до 30-40 м; сферичности частиц 0,8-1,0).

2. Проблема моделирования турбулентности все еще ждет своего решения и остается важной, особенно для течений смесей со сложной реофизической структурой, испытывающей влияние массовых сил, обусловленных закруткой стенки (при парамертрах от 0 до 60 об/мин). Анализ показывает, что возмущения от пульсационных процессов, нестабильность процессов переноса в дисперсных системах при сложных сдвиговых течениях $\left(\operatorname{Re}=(0,3-10) 10^{4}\right)$ вносит погрешности (от 4-5\%) в определение параметров локальной структуры при сравнении с имеющимися опытными данными. Это недопустимо при прогнозах реальных явлений и выдаче рекомендаций в практику приложений задач очистки. В частности, данные [1-3, $22,33,45,61,77,80]$ указывают, что модели типа вихревой вязкости весьма ограничены из-за невозможности реагировать на детали формирования течения с неоднородной турбулентностью при выраженном характере влияния центробежных и инерционных сил, определяемых вращением. Однако в условиях прямоточного и слабозакрученного течения гомогенной и гетерогенной смеси (малые значения критерия Россби: Ro<0,5 - турбулентные режимы; Ro $<2$ - ламинарный поток) прогнозы процессов по изменению перепада давления, концентраций смеси с использованием ке-, $\kappa \omega$ SST-моделей вполне пригодны. Для более широких условий течений в скважинах имеются лишь отдельные сведения прогноза их анизотропной структуры (например, [22, 33, 71, 73, 77, 80, 83]) и требуется разработка новых подходов и моделей [84-89].

3. Наибольшие проблемы расчета транспорта шламов связаны с моделированием процессов в дисперсной фазе. Установлено, что вполне оптимален с точки зрения затрат на реализацию и точность расчета эффектов течений эйлеров подход. При нем получены более точные результаты формирования полей динамического и диффузионного состава смеси, особенно при учете геометрических деталей о частицах (в рамках положений теории гранулярного течения) и эффектов межфазного взаимодействия.

4. Лагранжев подход, как более детальный, нуждается в гораздо больших вычислительных ресурсах, весьма чувствителен к заданию конфигурации и размеров частиц, их взаимодействий. В таком подходе на точность моделирования сильно влияет любая неопределенность данных, необходимых для формулировки краевых условий задачи (например, сведения о форме частиц). В рамках подхода обнаружены тонкие рециркуляционные эффекты, обусловленные вторичными течениями, для прогноза которых требуются соответствующие модели турбулентности более высокого порядка.

5. Преобладающими в процессах очистки скважин выступают эффекты, формируемые вращением внутренней трубы (вокруг своей оси - подвижная стенка; вокруг оси буровой колонны - орбитальное движение). Общий анализ результатов таких режимов показывает, что орбитальное движение внутренней трубы имеет определяющее значение в процессах очистки скважин в сравнении с потоком, испытывающим влияние от вращения внутренней трубы вдоль своей собственной оси. Изучение механизмов (например, согласно [77]), определяющих течение в данных условиях, способно уяснить физику процессов транспорта шламов, происходящих в забоях скважин.

6. Включение в модель технологических особенностей бурового оборудования (например, геометрии соединительных узлов между трубами) способно повысить точность прогноза процессов транспорта шлама из горизонтальных участков.

Анализ показывает, что перспективу дальнейших исследований составят задачи:

1. Разработки основ теории, объясняющих суть эффектов в процессах транспорта шламов из горизонтальных скважин с помощью идей эйлерова и лагранжева подходов, новых моделей турбулентности с дифференциальными уравнениями для корреляций пульсаций вектора скорости и скаляра. Это предмет гидродинамических задач по обоб- 
щению известных моделей для напряжений Рейнольдса на класс закрученных (по орбитальному типу) гетерогенных внутренних течений с современными двухпараметрическими базами.

2. Проведения исследований по установлению преимуществ отобранных моделей турбулентности для описания транспорта шламов при сложном сдвиговом потоке с учетом изменений значений параметров гранулярного состава и течения (например, угла между частицами, моделей гра-

\section{СПИСОК ЛИТЕРАТУРЫ}

1. Prospects of RANS models with multiparameter effects at simulation of complex non-isothermal flows of viscous media in devices with any configuration of surface / S.N. Kharlamov V.Yu. Kim, S.I. Silvestrov, R.A. Alginov, S.A. Pavlov // The $6^{t}$ Proceedings of the International Forum on Strategic Technology (IFOST-2010). - Harbin, China, August 22-24, 2011. - V. 2. P. 787-791.

2. Харламов С.Н., Фатьянов Д.М. Исследование структуры турбулентного потока природного сырья в трубопроводах с секцией переменного по длине поперечного сечения конфузорнодиффузорного типа // Известия Томского политехнического университета. Инжиниринг георесурсов. - 2020. - Т. 331. № 8. - C. 53-67.

3. Харламов С.Н., Джангхорбани М. Процессы транспорта шлама при очистке скважин с произвольной ориентацией буровых труб, содержащих эксцентрично расположенное круглое ядро с подвижной стенкой: проблемы, результаты, перспективы (обзор) // Известия Томского политехнического университета. Инжиниринг георесурсов. - 2020. - Т. 331. - № 7. - С. 131-149.

4. Pigott R.J.S. Mud flow in drilling // Drilling and production practice. - New York, USA, American Petroleum Institute, 1942. - P. 91-103.

5. Zeidler H.U. Fluid and drilled particle dynamics related to drilling mud carrying capacity. PhD thesis. - Oklahoma, USA, 1974. $304 \mathrm{p}$.

6. Thomas R.P., Azar J.J., Becker T.E. Drillpipe eccentricity effect on drilled cuttings behavior in vertical wellbores // Journal of Petroleum Technology. - 1982. - V. 34. - № 9. - P. 920-929.

7. Hussaini S.M., Azar J.J. Experimental study of drilled cuttings transport using common drilling muds // Society of Petroleum Engineers Journal. - 1983. - V. 23. - № 1. - P. 11-20.

8. Qureshi M.A. Experimental study on effective hole cleaning using mechanical cleaning devices. MS Thesis. - Oklahoma, USA, 2004. $-180 \mathrm{p}$.

9. Okrajni S., Azar J.J. The effects of mud rheology on annular hole cleaning in directional wells // SPE Drilling Engineering. - 1986. V. 1. - № 4. - P. 297-308.

10. Review and analysis of cuttings transport in complex structural wells / W. Kelin, Y. Tie, S. Xiaofeng, S. Shuai, L. Shizhu // The Open Fuels \& Energy Science Journal. - 2013. - V. 6. - № 1. P. 9-17.

11. Lummus James L., Azar J.J. Drilling fluids optimization: a practical field approach. - Tulsa, PennWell, USA, 1986. - 196 p.

12. Kelessidis V.C., Hatzistamou V. Preparation methodology and rheological properties of yield pseudoplastic transparent fluids // Journal of Dispersion science and Technology. - 2011. - V. 32. № 3. - P. 380-388.

13. Nazari T., Hareland G., Azar J.J. Review of cuttings transport in directional well drilling: systematic approach // SPE Western Regional Meeting. - Anaheim, California, USA: Society of Petroleum Engineers, 2010. - P. 1-15.

14. Wadell Hakon. Volume, shape and roundness of quartz particles // The Journal of Geology. - 1935. - V. 43. - № 3. -P. 250-280.

15. Drill cutting transport in full scale vertical annuli / T.R. Sifferman, G.M. Myers, E.L. Haden, H.A. Wahl // Fall Meeting of the Society of Petroleum Engineers of AIME. - Las Vegas, Nevada, USA: Society of Petroleum Engineers, 1973. - 12 p.

16. Kharlamov S.N. Heat and mass transfer in facilities with a moving piston // Heat Transfer Research. - 2007. - V. 38. - № 3. P. 233-243. нулярной вязкости и т. д.) при эйлеровом и лагранжевом подходах.

3. Детального моделирования процессов гидродинамики и тепломассопереноса в межтрубном пространстве при течении смеси с заданной формой частиц, оценок преимуществ лагранжева подхода, верификации результатов по опытным данным, полученным на основе современной техники (позитронной эмиссионной томографии, бесконтактной регистрации).

17. Shook C.A., Roco M.C. Slurry flow: principles and practice. London, UK: Butterworth-Heimemann, 1991. - 324 p.

18. Kharlamov S.N., Kim V.Yu., Silvestrov S.I. Numerical modelling of a vortical investigation of heat transfer in fields of centrifugal mass forces in elements of the power equipment with a curvilinear wall // The $5^{\text {th }}$ Proceedings of the International Forum on Strategic Technology (IFOST-2010). - Ulsan, Korea, October 13-15, 2010 - P. 105-109.

19. Nigmatulin R.I., Khabeev N.S., Nagiev F.B. Dynamics, heat and mass transfer of vapour-gas bubbles in a liquid // International Journal of Heat and Mass Transfer. - 1981. - V. 24. - № 6. P. $1033-1041$

20. Sommerfed M. Theoretical and experimental modeling of particulate flows: overview and fundamentals. P. I-II. Lectures Series 2000-6. - Karlsruhe, Germany: Von Karman Institute for Fluid Dynamics, 2000. $-60 \mathrm{p}$.

21. Crowe C.T., Sommerfeld M., Tsuji Y. Fundamentals of gasparticle and gas-droplet flows. - Boca Raton, USA: CRCPress, 1998. $-179 \mathrm{p}$

22. A study on the validity of the multi-sphere discrete element method / H. Kruggel-Emdem, S. Rikelt, S. Wirtz, V. Scherer // Powder Technology. - 2008. - V. 188. - № 2. - P. 153-165.

23. Akhshik S., Behzad M., Rajabi M. CFD-DEM simulation of the hole cleaning process in a deviated well drilling: the effects of particle shape // Particuology. - 2016. - V. 25. - P. 72-82.

24. Lain S., Sommerfeld M. Euler/Lagrange computations of pneumatic conveying in a horizontal channel with different wall roughness // Powder Technology. - 2008. - V. 184. - P. 76-88.

25. Mei K. An approximate expression for the shear lift force on a spherical particle at finite Reynolds number // International Journal of Multiphase Flow. - 1992. - V. 18. - № 1. - P. 145-147.

26. Kuang S.B., Yu A.B., Zou Z.S. Computational study of flow regimes in vertical pneumatic conveying // Industrial and Engineering Chemistry Research. - 2009. - V. 48. - № 14. P. 6846-6858.

27. Di Renzo A., Di Maio F.P. Comparison of contact-force models for the simulation of collisions in DEM-based granular flow codes // Chemical Engineering Science. - 2004. - V. 59. - № 3. - P. 525-541.

28. Chien S.F. Settling velocity of irregularly shaped particles // SPE Drilling and Completion. - 1994. - V. 9. - № 4. - P. 281-289.

29. Flow instabilities of Herschel-Bulkey fluids / A.N. Alexandrou, P. Le Menn, G. Georgiou, V. Entov // Journal of Non-Newtonian Fluid Mechanics. - 2003. - V. 116. - № 1. - P. 19-32.

30. Saffman P.G. The lift on a small sphere in a slow shear flow // Journal of Fluid Mechanics. - 1965. - V. 22 - № 2. - P. 385-400.

31. Oesterle B., Bui D.T. Experiments on the lift of a spinning sphere in a range of intermediate Reynolds numbers // Experiments in fluids. -1998 . - V. 25. - № 1. - P. 16-22.

32. Computational Fluid Dynamics (CFD) as a tool to study cutting transport in wellbores / H.I. Bilgesu, M.W. Ali, K. Aminian, S. Ameri // The Eastern Regional Meeting of the Society of Petroleum Engineers. - Lexington, Kentucky, USA, October 2002. $27 \mathrm{p}$.

33. Mishra Nekkhil. Investigation of hole cleaning parameters using computational fluid dynamics in horizontal and deviated wells. Graduate Theses. - USA, 2007. $-76 \mathrm{p}$.

34. CFD simulation of single-phase two-phase flow in gas-liquid cylindrical cyclone separators. Paper SPE 36645 / F.M. Erdal, S.A. Shirazi, O. Shoham, G.E. Kouba // SPE Annual Technical Conference and Exhibition. - Denver, CO, October 6-9, 1996. P. $1-15$. 
35. Solid-liquid hydrodynamics in a slim hole drilling annulus S.M. Han, Y.K. Hwang, N.S. Woo, Y.J. Kim // Journal of Petroleum Science and Engineering. - 2010. - V. 70. - № 3-4. - P. 308-319.

36. Saggot A.M., Dupuis D.C. A major step in ultra slimhole drilling // SPE Annual Technical Conference. - New Orleans, September 1994. $-10 \mathrm{p}$.

37. Simulation of the cuttings cleaning during the drilling operation / H.H. Al-Kayiem, M.Z.A. Ismail, N.M. Zaki, M.E. Elfeel // American Journal of Applied Sciences. - 2010. - V. 7. - № 6. - P. 800-806.

38. Pereira F.A.R., Ataíde C.H., Barrozo M.A.S. CFD Approach using a discrete phase model for annular flow analysis // Latin American applied research. - 2010. - V. 40. - № 1. - P. 53-60.

39. Launder B.E., Spalding D.B. The numerical computation of turbulent flows // Numerical prediction of flow, heat transfer turbulence and combustion. - London: Pergamon, 1983. - P. 96-116.

40. Mme U., Skalle P. CFD calculations of cuttings transport through drilling annuli at various angles // International Journal of Petroleum Science and Technology. - 2012. - V. 6. - № 2. P. 129-141.

41. Computational modeling of drilling fluids dynamics in casing drilling / M. Mokhtari, M.A. Ermila, A.N. Tutuncu, M. Karimi / SPE Eastern Regional Meeting. - Lexington, Kentucky, USA October 2012. - P. 1-13.

42. Zhu X.H., Sun C., Tong H. Distribution features, transport mechanism and destruction of cuttings bed in horizontal well // Journal of Hydrodynamics. - 2013. - V. 25. - № 4. - P. 628-638.

43. Osgouei R.E., Ozbayoglu M.E., Fu T.K. CFD simulation of solids carrying capacity of a newtonian fluid through horizontal eccentric annulus // ASME 2013 Fluids Engineering Division Summer Meeting. - Incline Village, Nevada, USA, July 2013. - P. 1-9.

44. Gas-liquid flow through horizontal eccentric annuli: CFD and experiments compared / M. Sorgun, R.E. Osgouei, M.E Ozbayoglu, A.M. Ozbayoglu // ASME-JSME-KSME 2011 Joint Fluids Engineering Conference. - Hamamatsu, Shizuoka, Japan, January, 2011. - P. 3847-3852.

45. Demiralp Y. Effects of drill-pipe whirling motion on cuttings transport performance for horizontal drilling. Master dissertation. - USA, 2014. - $151 \mathrm{p}$.

46. Menter F.R. Two-equation eddy-viscosity turbulence models for engineering applications // AIAA journal. - 1994. - V. 32. № 8. - P. 1598-1605.

47. Cundall P.A., Strack O.D. A discrete numerical model for granular assemblies // Geotechnique. - 1979. - V. 29. - № 1. - P. 47-65.

48. Determination of cuttings lag in horizontal and deviated wells A.J. Garcia-Hernandez, S.Z. Miska, M. Yu, N.E. Takach, C.M. Zettner // SPE Annual Technical Conference and Exhibition. - Anaheim, California, USA, November, 2017. - 11 p.

49. Wilcox D.C. Turbulence modeling for CFD. - La Canada, California, USA, 1998. - V. 2. - 461 p.

50. Simulation of cuttings transport with foam in deviated wellbores using computational fluid dynamics / R. Rooki, F.D. Ardejani, A. Moradzadeh, M. Norouzi // Journal of Petroleum Exploration and Production Technology. - 2014. - V. 4. - № 3. - P. 263-273.

51. CFD simulation of rheological model effect on cuttings transport / R. Rooki, F.D. Ardejani, A. Moradzadeh, M. Norouzi // Journal of Dispersion Science and Technology. - 2015. - V. 36. - № 3. P. 402-410.

52. Beyer A.H., Millhone R.S., Foote R.W. Flow behavior of foam as a well circulating fluid // Proceedings of the Society of Petroleum Engineers of AIME. - San Antonio, Texas, USA, 1972. - P. 1-12.

53. Cuttings transport with foam in horizontal \& highly-inclined wellbores / E.M. Ozbayoglu, S.Z. Miska, T. Reed, N. Takach // SPE/IADC Drilling Conference. - Amsterdam, the Netherlands, January 2003. - P. 1-9.

54. Bonilla L.F., Shah S.N. Experimental investigation on the rheology of foams // Proceedings of the SPE/CERI Gas Technology Symposium. - Calgary, Canada, April 2000. - P. 1-14.

55. Ofei T.N., Pao W. Modelling of pressure drop and cuttings concentration in eccentric narrow horizontal wellbore with rotating drillpipe // Journal of Applied Sciences. - 2014. - V. 14. - № 23. P. 3263-3269.

56. Ofei T.N., Irawan S., Pao W. CFD method for predicting annular pressure losses and cuttings concentration in eccentric horizontal wells // Journal of Petroleum Engineering. - 2014. - V. 115. - P. 1-16.
57. Launder B.E., Dudley B.S. Mathematical models of turbulence // Journal of Fluid Mechanics. - 1973. - V. 57. - Iss. 4. - № 6. P. 826-828.

58. Ofei T.N., Alhemyari S.A. Computational fluid dynamics simulation of the effect of drill pipe rotation on cuttings transport in horizontal wellbores using a Newtonian fluid // International Field Exploration and Development Conference. - Xian, China, September 2015. - P. 8-16.

59. Ofei T.N. Effect of yield power law fluid rheological properties on cuttings transport in eccentric horizontal narrow annulus // Journal of Fluids. - 2016. - V. 139. - P. 11-22.

60. Kamyab M., Rasouli V. Experimental and numerical simulation of cuttings transportation in coiled tubing drilling // Journal of Natural Gas Science and Engineering. - 2016. - V. 29. - P. 284-302.

61. Akhshik S., Rajabi M. CFD-DEM modeling of cuttings transport in underbalanced drilling considering aerated mud effects and downhole conditions // Journal of Petroleum Science and Engineering. - 2018. - V. 160. - P. 229-246.

62. Cuttings transport in directional and horizontal wells while aerated mud drilling / S. Naganawa, A. Oikawa, Y. Masuda, Y. Tetsuo, M. Hoshino, P. Acuna // IADC/SPE Asia Pacific Drilling Technology. - Jakarta, Indonesia, September, 2002. - P. 11-20.

63. Barigou M. Particle tracking in opaque mixing systems: an overview of the capabilities of PET and PEPT // Chemical Engineering Research and Design. - 2004. - V. 82. - № 9. P. $1258-1267$.

64. Epelle E.I., Gerogiorgis D.I. Transient and steady state analysis of drill cuttings transport phenomena under turbulent conditions // Chemical Engineering Research and Design. - 2018. - V. 131. P. 520-544.

65. Epelle E.I., Gerogiorgis D.I. CFD modelling and simulation of drill cuttings transport efficiency in annular bends: effect of particle sphericity // Journal of Petroleum Science and Engineering. - 2018. - V. 170. - P. 992-1004.

66. Syamlal Madhava, O'Brien Th.J. Computer simulation of bubbles in a fluidized bed // American Institute of Chemical Engineers. 1989. - V. 85. - № 1. - P. 22-31.

67. Sorgun M. Modeling of Newtonian fluids and cuttings transport analysis in high inclination wellbores with pipe rotation. MSc Thesis. - Ankara, Turkey, 2010. - 177 p.

68. Tomren P.H., Iyoho A.W., Azar J.J. Experimental study of cuttings transport in directional wells // SPE Drilling Engineering. - 1986. - V. 1. - № 1. - P. 43-56.

69. Hajipour Mastaneh. CFD simulation of turbulent flow of drill cuttings and parametric studies in a horizontal annulus // Scientific Notes. Applied Sciences. - 2020. - V. 2. - P. 1-12.

70. A study on non-spherical cuttings transport in CBM well drilling by coupled CFD-DEM / Shao Bing, Yifei Yan, Xiangzhen Yan, Zhiqian $\mathrm{Xu} / /$ Engineering Applications of Computational Fluid Mechanics. - 2019. - V. 13. - № 1. - P. 579-590.

71. CFD modeling of hydraulic behavior of oil-and water-based drilling fluids in laminar flow / S. Sayindla, B. Lund, J.D. Ytrehus, A. Saasen // SPE Drilling \& Completion. - 2019. - V. 34. Iss. 3. - P. 171-180.

72. Kelessidis V.C., Dalamarinis P., Maglione R. Experimental study and predictions of pressure losses of fluids modeled as HerschelBulkley in concentric and eccentric annuli in laminar, transitional and turbulent flows // Journal of Petroleum Science and Engineering. - 2011. - V. 77. - № 3-4. - P. 305-312.

73. Heydari O., Sahraei E., Skalle P. Investigating the impact of drillpipe's rotation and eccentricity on cuttings transport phenomenon in various horizontal annuluses using computational fluid dynamics (CFD) // Journal of Petroleum Science and Engineering. - 2017. - V. 156. - P. 801-813.

74. Transport of small cuttings in Exended Reach Drilling / M. Duan, S. Miska, M. Yu, N.E. Takach, R.M. Ahmed, J.H. Hallman // SPE Oil and Gas Conference and Exhibition. - Beijing, China, December 2006. - P. 1-9.

75. Gidaspow D. Multiphase flow and fluidization: continuum and kinetic theory descriptions. - London, UK: Academic press, 1994. - $461 \mathrm{p}$.

76. Chen Zhu. Experimental study on cuttings transport with foam under simulated horizontal downhole conditions // SPE Drilling \& Completion. - 2007. - V. 22. - № 4. - P. 304-312.

77. Experimental study and modeling of cuttings transport using foam with drillpipe rotation / M. Duan, S. Miska, M. Yu, N.E. Takach, 
R.M. Ahmed, J.H. Hallman // SPE Drilling \& completion. 2010. - V. 25. - № 3. - P. 352-362.

78. Steady-state cuttings transport simulation in horizontal borehole annulus / Y. Ignatenko, O. Bocharov, A. Gavrilov, R. May // ASME $201837^{\text {th }}$ International Conference on Ocean, Offshore and Arctic Engineering. - Madrid, Spain, June 2018. - P. 26-38.

79. Miller R.M., Morris, J.F. Normal stress driven migration and axial development in pressure-driven flow of concentrated suspensions // Journal of Non-Newtonian Fluid Mechanics. - 2006. - V. 135. P. $149-165$.

80. Johnson P.C., Jackson R. Frictional collisional constitutive relations for granular materials with application of plane shearing // Journal of Fluid Mechanics. - 1987. - V. 176. - № 3. - P. 67-93.

81. Effect of orbital motion of drill pipe on the transport of nonNewtonian fluid-cuttings mixture in horizontal drilling annulus / B. Pang, S. Wang, X. Jiang, H. Lu // Journal of Petroleum Science and Engineering. - 2019. - V. 174. - P. 201-215.

82. Miska S., Reed T., Kuru E. Advanced cuttings transport study: final technical report (date of issue: September 30 2004, DOE Award number: DE-FG26-99 BC15178. The University of Tulsa, 600 South College Avenue, Tulsa, Oklahoma 74104). - Tulsa, Oklahoma, 2004. -284 p.

83. Busch A., Johansen S.T. Cuttings transport: on the effect of drill pipe rotation and lateral motion on the cuttings bed // Journal of Petroleum Science and Engineering. - 2020. - V. 191. - P. 107-136.

84. Investigation of cuttings transport in directional and horizontal drilling wellbores injected with pulsed drilling fluid using CFD approach / B. Pang, S. Wang, C. Lu, W. Cai, X. Jiang, H. Lu // Tunnelling and Underground Space Technology. - 2019. V. 90. - P. 183-193.

85. Numerical investigation of a double-circulation system for cuttings transport in CBM well drilling using a CFD-DEM coupled model / B. Shao, Y. Yan, X. Wang, F. Liao, X. Yan // Engineering Applications of Computational Fluid Mechanics. - 2020. V. 14. - № 1. - P. 38-52.

86. Al-Gailani A., Al-Yasiri M., Wen D. Numerical study of cuttings transport of nanoparticle-based drilling fluid // Engineering Reports. - 2020. - № (e). - P. 12154-12168.

87. Escudier M.P., Nickson A.K., Poole R.J. Turbulent flow of viscoelastic shear-thinning liquids through a rectangular duct: quantification of turbulence anisotropy // Journal of nonnewtonian fluid mechanics. - 2009. - V. 160. - P. 2-10.

88. Systematic sensitivity analysis of cuttings transport in drilling operation using computational fluid dynamics approach / M.M. Huque, S. Butt, S. Zendehboudi, S. Imtiaz // Journal of Natural Gas Science and Engineering. - 2020. - V. 81. № 103386. - P. 17-38.

89. Modeling of dynamic cuttings transportation during drilling of oil and gas wells by combining 2D CFD and 1D discretization approach / F. Zhang, Y. Wang, Y. Wang, S. Miska, M. Yu // SPE Journal. - 2020. - V. 25. - Iss. 3. - № 6. - P. 21-42.

Поступила 19.04.2021 г.

\section{Информация об авторах}

Харламов С.Н., доктор физико-математических наук, профессор, профессор отделения нефтегазового дела Инженерной школы природных ресурсов Национального исследовательского Томского политехнического университета.

Джжагхорбани М., аспирант отделения нефтегазового дела Инженерной школы природных ресурсов Национального исследовательского Томского политехнического университета.

Филиппов К.A., аспирант отделения нефтегазового дела Инженерной школы природных ресурсов Национального исследовательского Томского политехнического университета. 
UDC 532.517:536.242:544.012:622.244:519.6

\title{
MATHEMATICAL MODELING AND RESEARCH METHODS OF HORIZONTAL WELLS HYDRODYNAMIC CLEANING
}

\author{
Sergey N. Kharlamov 1 , \\ kharsn@mail.ru
}

\author{
Janghorbani Mehran', \\ mehran.janghorbani@gmail.com
}

\author{
Konstantin A. Filippov ${ }^{1}$, \\ kost.filippow1@yandex.ru \\ 1 National Research Tomsk Polytechnic University, \\ 30, Lenin avenue, Tomsk, 634050, Russia.
}

\begin{abstract}
Relevance of the research is determined by the need to understand modern achievements in modeling the processes of sludge transport in real conditions of drilling operations that increase drilling speed and the efficiency of well cleaning; to establish the advantages/disadvantages of existing approaches, modern models and complex methods for describing the dynamics of systems with particles in order to effectively choose the rheological properties of drilling fluids and eliminate the reasons of equipment loss.

The aim of this work is to understand the modern experience of modeling the processes of cleaning wells with complex geometries, mainly lying in the horizontal plane; to study the features of the flow of mixtures in the annular space with a circular core; providing recommendations for the practice of modeling and calculating hydrodynamic processes that increase hole cleaning efficiency.

Methodology. Theoretical and practical research methods from related fields of hydrodynamics and heat and mass transfer in rheologicalIy and physically complex systems; methods for numerical modeling of laminar and turbulent flows under conditions of direct-flow and swirling flows created by a co-axially or orbitally rotating wall, which affect the formation of a layer of cuttings and the dynamics of particles of a dispersed mixture.

Results. The paper introduces the results of modern numerical studies of cleaning wells with horizontal sections and the information on aspects of detailed hydrodynamic and diffusion modeling of complex flows in inclined wells. The applied models and methods used to study the characteristics of flow and heat and mass transfer in homogeneous and heterogeneous media in internal systems are formulated. Aspects of modeling the processes of cuttings transport in the framework of Euler/Lagrangian approaches are discussed, taking into account the features introduced by the effects of interfacial interaction/rotation. The paper introduces the changes in cuttings concentration based on rheological properties (power-law or Herschel-Bulkley), different shapes/sizes of particles, flow regimes and inertial forces. The problems of improving the accuracy of cuttings transport modeling, the possibilities of new technologies, original turbulence models and their two-parameter dynamic bases used for increasing the intensification and efficiency of cleaning processes are analyzed. The authors have given the recommendations for their solution in the considered hydrodynamic and geometric configurations. The results and problems that are of fundamental and applied significance and constitute an independent subject of prospective research are indicated.
\end{abstract}

\section{Key words:}

Cuttings, well, cleaning, modeling, hydrodynamics, mass transfer, rheology, turbulence, structure, forces.

\section{REFERENCES}

1. Kharlamov S.N., Kim V.Yu., Silvestrov S.I., Alginov R.A., Pavlov S.A. Prospects of RANS models with multiparameter effects at simulation of complex non-isothermal flows of viscous media in devices with any configuration of surface. The $6^{\text {th }}$ Proceedings of the International Forum on Strategic Technology (IFOST-2010). Harbin, China, August 22-24, 2011. Vol. 2, pp. 787-791.

2. Kharlamov S.N., Fatyanov D.S. Investigation of natural raw materials turbulent flow structure in pipelines at confuser-diffuser section. Bulletin of the Tomsk Polytechnic University. Geo Assets Engineering, 2020, vol. 331, no. 8, pp. 53-67. In Rus.

3. Kharlamov S.N., Janghorbani M. Cuttings transport in hole cleaning considering well orientation, pipe eccentricity and pipe rotation: Problems, results, prospects (survey). Bulletin of the Tomsk Polytechnic University. Geo Assets Engineering, 2020, vol. 331, no. 7, pp. 131-149. In Rus.

4. Pigott R.J.S. Mud flow in drilling. Drilling and production practice New York, USA, American Petroleum Institute, 1942. pp. 91-103.

5. Zeidler H.U. Fluid and drilled particle dynamics related to drilling mud carrying capacity. $\mathrm{PhD}$ thesis. Oklahoma, USA, 1974. $304 \mathrm{p}$.

6. Thomas R.P., Azar J.J., Becker T.E. Drillpipe eccentricity effect on drilled cuttings behavior in vertical wellbores. Journal of Petroleum Technology, 1982, vol. 34, no. 9, pp. 920-929.

7. Hussaini S.M., Azar J.J. Experimental study of drilled cuttings transport using common drilling muds. Society of Petroleum Engineers Journal, 1983, vol. 23, no. 1, pp. 11-20.
8. Qureshi M.A. Experimental study on effective hole cleaning using mechanical cleaning devices. MS Thesis. Oklahoma, USA. 2004. $180 \mathrm{p}$.

9. Okrajni S., Azar J.J. The effects of mud rheology on annular hole cleaning in directional wells. SPE Drilling Engineering, 1986, vol. 1, no. 4, pp. 297-308.

10. Kelin W., Tie Y., Xiaofeng S., Shuai S., Shizhu L. Review and analysis of cuttings transport in complex structural wells. The Open Fuels \& Energy Science Journal, 2013, vol. 6, no. 1, pp. 9-17.

11. Lummus James L., Azar J.J. Drilling fluids optimization: a practical field approach. Tulsa, PennWell, USA, 1986. $196 \mathrm{p}$.

12. Kelessidis V.C., Hatzistamou V. Preparation methodology and rheological properties of yield pseudoplastic transparent fluids. Journal of Dispersion science and Technology, 2011, vol. 32, no. 3, pp. 380-388.

13. Nazari T., Hareland G., Azar J.J. Review of cuttings transport in directional well drilling: systematic approach. SPE Western Regional Meeting. Society of Petroleum Engineers. Anaheim, California, USA, May, 2010. pp. 1-15.

14. Wadell Hakon. Volume, shape and roundness of quartz particles. The Journal of Geology, 1935, vol. 43, no. 3, pp. 250-280.

15. Sifferman T.R., Myers G.M., Haden E.L., Wahl H.A. Drill cutting transport in full scale vertical annuli. Fall Meeting of the Society of Petroleum Engineers of AIME. Las Vegas, Nevada, USA, Society of Petroleum Engineers, Oct. 1973. 12 p.

16. Kharlamov S.N. Heat and mass transfer in facilities with a moving piston. Heat Transfer Research, 2007, vol. 38. no. 3, pp. 233-243. 
17. Shook C.A., Roco M.C. Slurry flow: principles and practice. London, UK, Butterworth-Heimemann, 1991. 324 p.

18. Kharlamov S.N., Kim V.Yu., Silvestrov S.I. Numerical modelling of a vortical investigation of heat transfer in fields of centrifugal mass forces in elements of the power equipment with a curvilinear wall. The $5^{\text {th }}$ Proceedings of the International Forum on Strategic Technology (IFOST-2010). Ulsan, Korea, October 13-15, 2010, pp. $105-109$.

19. Nigmatulin R.I., Khabeev N.S., Nagiev F.B. Dynamics, heat and mass transfer of vapour-gas bubbles in a liquid. International Journal of Heat and Mass Transfer, 1981, vol. 24, no. 6, pp. 1033-1041.

20. Sommerfed M. Theoretical and experimental modeling of particulate flows: overview and fundamentals. P. I-II. Lectures Serie 2000-6. Karlsruhe, Germany, Von Karman Institute for Fluid Dynamics, $2000.60 \mathrm{p}$

21. Crowe C.T., Sommerfeld M., Tsuji Y. Fundamentals of gasparticle and gas-droplet flows. Boca Raton, USA, CRCPress, $1998.179 \mathrm{p}$.

22. Kruggel-Emdem H., Rikelt S., Wirtz S., Scherer V. A study on the validity of the multi-sphere discrete element method. Powder Technology, 2008, vol. 188, no. 2, pp. 153-165.

23. Akhshik S., Behzad M., Rajabi M. CFD-DEM simulation of the hole cleaning process in a deviated well drilling: the effects of particle shape. Particuology, 2016, vol. 25, pp. 72-82.

24. Lain S., Sommerfeld M. Euler/Lagrange computations of pneumatic conveying in a horizontal channel with different wall roughness. Powder Technology, 2008, vol. 184, pp. 76-88.

25. Mei K. An approximate expression for the shear lift force on a spherical particle at finite Reynolds number. International Journal of Multiphase Flow, 1992, vol. 18, no. 1, pp. 145-147.

26. Kuang S.B., Yu A.B., Zou Z.S. Computational study of flow regimes in vertical pneumatic conveying. Industrial and Engineering Chemistry Research, 2009, vol. 48, no. 14, pp. 6846-6858

27. Di Renzo A., Di Maio F.P. Comparison of contact-force models for the simulation of collisions in DEM-based granular flow codes. Chemical Engineering Science, 2004, vol. 59, no. 3, pp. 525-541.

28. Chien S.F. Settling velocity of irregularly shaped particles. SPE Drilling and Completion, 1994, vol. 9, no. 4, pp. 281-289.

29. Alexandrou A.N., Le Menn P., Georgiou G., Entov V. Flow instabilities of Herschel-Bulkey fluids. Journal of Non-Newtonian Fluid Mechanics, 2003, vol. 116, no. 1, pp. 19-32.

30. Saffman P.G. The lift on a small sphere in a slow shear flow. Journal of Fluid Mechanics, 1965, vol. 22, no. 2, pp. 385-400.

31. Oesterle B., Bui D.T. Experiments on the lift of a spinning sphere in a range of intermediate Reynolds numbers. Experiments in fluids, 1998, vol. 25, no. 1, pp. 16-22.

32. Bilgesu H.I., Ali M.W., Aminian K., Ameri S. Computational Fluid Dynamics (CFD) as a tool to study cutting transport in wellbores. The Eastern Regional Meeting of the Society of Petroleum Engineers. Lexington, Kentucky, USA, October 2002. 27 p.

33. Mishra Nekkhil. Investigation of hole cleaning parameters using computational fluid dynamics in horizontal and deviated wells. Graduate Theses. USA, 2007.76 p.

34. Erdal F.M., Shirazi S.A., Shoham O., Kouba G.E. CFD simulation of single-phase two-phase flow in gas-liquid cylindrical cyclone separators. Paper SPE 36645. SPE Annual Technical Conference and Exhibition. Denver, CO, October 6-9, 1996. pp. 1-15.

35. Han S.M., Hwang Y.K., Woo N.S., Kim Y.J. Solid-liquid hydrodynamics in a slim hole drilling annulus. Journal of Petroleum Science and Engineering, 2010, vol. 70, no. 3-4, pp. 308-319.

36. Saggot A.M., Dupuis D.C. A major step in ultra slimhole drilling. SPE Annual Technical Conference. New Orleans, September 1994.10 p.

37. Al-Kayiem H.H., Ismail M.Z.A., Zaki N.M., Elfeel M.E. Simulation of the cuttings cleaning during the drilling operation. American Journal of Applied Sciences, 2010, vol. 7, no. 6, pp. 800-806.

38. Pereira F.A.R., Ataíde C.H., Barrozo M.A.S. CFD Approach using a discrete phase model for annular flow analysis. Latin American applied research, 2010, vol. 40, no. 1, pp. 53-60.

39. Launder B.E., Spalding D.B. The numerical computation of turbulent flows. Numerical prediction of flow, heat transfer, turbulence and combustion. London, Pergamon, 1983. pp. 96-116.

40. Mme U., Skalle P. CFD calculations of cuttings transport through drilling annuli at various angles. International Journal of Petroleum Science and Technology, 2012, vol. 6, no. 2, pp. 129-141.
41. Mokhtari M., Ermila M.A., Tutuncu A.N., Karimi M. Computational modeling of drilling fluids dynamics in casing drilling. SPE Eastern Regional Meeting. Lexington, Kentucky, USA, October 2012. pp. 1-13.

42. Zhu X.H., Sun C., Tong H. Distribution features, transport mechanism and destruction of cuttings bed in horizontal well. Journal of Hydrodynamics, 2013, vol. 25, no. 4, pp. 628-638.

43. Osgouei R.E., Ozbayoglu M.E., Fu T.K. CFD simulation of solids carrying capacity of a newtonian fluid through horizontal eccentric annulus. ASME 2013 Fluids Engineering Division Summer Meeting. Incline Village, Nevada, USA, July 2013, pp. 1-9.

44. Sorgun M., Osgouei R.E., Ozbayoglu M.E., Ozbayoglu A.M. Gasliquid flow through horizontal eccentric annuli: CFD and experiments compared. ASME-JSME-KSME 2011 Joint Fluids Engineering Conference. Hamamatsu, Shizuoka, Japan, January 2011, pp. 3847-3852.

45. Demiralp Y. Effects of drill-pipe whirling motion on cuttings transport performance for horizontal drilling. Master dissertation. USA, 2014. $151 \mathrm{p}$

46. Menter F.R. Two-equation eddy-viscosity turbulence models for engineering applications. AIAA journal, 1994, vol. 32, no. 8, pp. $1598-1605$.

47. Cundall P.A., Strack O.D. A discrete numerical model for granular assemblies. Geotechnique, 1979, vol. 29, no. 1, pp. 47-65.

48. Garcia-Hernandez A.J., Miska S.Z., Yu M., Takach N.E., Zettner C.M. Determination of cuttings lag in horizontal and deviated wells. SPE Annual Technical Conference and Exhibition. Anaheim, California, USA, November 2017. $11 \mathrm{p}$.

49. Wilcox D.C. Turbulence modeling for CFD. La Canada, California, USA, 1998. Vol. 2, 461 p.

50. Rooki R., Ardejani F.D., Moradzadeh A., Norouzi M. Simulation of cuttings transport with foam in deviated wellbores using computational fluid dynamics. Journal of Petroleum Exploration and Production Technology, 2014, vol. 4, no. 3, pp. 263-273.

51. Rooki R., Ardejani F.D., Moradzadeh A., Norouzi M. CFD simulation of rheological model effect on cuttings transport. Journal of Dispersion Science and Technology, 2015, vol. 36, no. 3, pp. 402-410.

52. Beyer A.H., Millhone R.S., Foote R.W. Flow behavior of foam as a well circulating fluid, Proceedings of the Society of Petroleum Engineers of AIME. San Antonio, Texas, USA, 1972. pp. 1-12.

53. Ozbayoglu E.M., Miska S.Z., Reed T., Takach N. Cuttings transport with foam in horizontal \& highly-inclined wellbores. SPE/IADC Drilling Conference. Amsterdam, the Netherlands, January 2003. pp. 1-9.

54. Bonilla L.F., Shah S.N. Experimental investigation on the rheology of foams. Proceedings of the SPE/CERI Gas Technology Symposium. Calgary, Canada, April 2000. pp. 1-14.

55. Ofei T.N., Pao W. Modelling of pressure drop and cuttings concentration in eccentric narrow horizontal wellbore with rotating drillpipe. Journal of Applied Sciences, 2014, vol. 14, no. 23, pp. 3263-3269.

56. Ofei T.N., Irawan S., Pao W. CFD method for predicting annular pressure losses and cuttings concentration in eccentric horizontal wells. Journal of Petroleum Engineering, 2014, vol. 115, pp. 1-16.

57. Launder B.E., Dudley B.S. Mathematical models of turbulence. Journal of Fluid Mechanics, 1973, vol. 57, Iss. 4, no. 6, pp. 826-828.

58. Ofei T.N., Alhemyari S.A. Computational fluid dynamics simulation of the effect of drill pipe rotation on cuttings transport in horizontal wellbores using a Newtonian fluid. International Field Exploration and Development Conference. Xian, China, September 2015. pp. 8-16.

59. Ofei T.N. Effect of yield power law fluid rheological properties on cuttings transport in eccentric horizontal narrow annulus. Journal of Fluids, 2016, vol.139, pp. 11-22.

60. Kamyab M., Rasouli V. Experimental and numerical simulation of cuttings transportation in coiled tubing drilling. Journal of Natural Gas Science and Engineering, 2016, vol. 29, pp. 284-302.

61. Akhshik S., Rajabi M. CFD-DEM modeling of cuttings transport in underbalanced drilling considering aerated mud effects and downhole conditions. Journal of Petroleum Science and Engineering, 2018, vol. 160, pp. 229-246.

62. Naganawa S., Oikawa A., Masuda Y., Tetsuo Y., Hoshino M., Acuna P. Cuttings transport in directional and horizontal wells while aerated mud drilling. IADC/SPE Asia Pacific Drilling Technology. Jakarta, Indonesia, September, 2002. pp. 11-20. 
63. Barigou M. Particle tracking in opaque mixing systems: an overview of the capabilities of PET and PEPT. Chemical Engineering Research and Design, 2004, vol. 82, no. 9, pp. 1258-1267.

64. Epelle E.I., Gerogiorgis D.I. Transient and steady state analysis of drill cuttings transport phenomena under turbulent conditions. Chemical Engineering Research and Design, 2018, vol. 131, pp. 520-544.

65. Epelle E.I., Gerogiorgis D.I. CFD modelling and simulation of drill cuttings transport efficiency in annular bends: effect of particle sphericity. Journal of Petroleum Science and Engineering, 2018, vol. 170, pp. 992-1004.

66. Syamlal Madhava, O'Brien Th.J. Computer simulation of bubbles in a fluidized bed. American Institute of Chemical Engineers, 1989 , vol. 85 , no. 1, pp. $22-31$.

67. Sorgun M. Modeling of Newtonian fluids and cuttings transport analysis in high inclination wellbores with pipe rotation. MSc Thesis. Ankara, Turkey, 2010. $177 \mathrm{p}$

68. Tomren P.H., Iyoho A.W., Azar J.J. Experimental study of cuttings transport in directional wells. SPE Drilling Engineering, 1986, vol. 1, no. 1, pp. 43-56.

69. Hajipour Mastaneh. CFD simulation of turbulent flow of drill cuttings and parametric studies in a horizontal annulus. Scientific Notes. Applied Sciences, 2020, vol. 2, pp. 1-12.

70. Shao Bing, Yifei Yan, Xiangzhen Yan, Zhiqian Xu. A study on non-spherical cuttings transport in CBM well drilling by coupled CFD-DEM. Engineering Applications of Computational Fluid Mechanics, 2019, vol. 13, no. 1, pp. 579-590.

71. Sayindla S., Lund B., Ytrehus J. D., Saasen A. CFD modeling of hydraulic behavior of oil-and water-based drilling fluids in lamina flow. SPE Drilling \& Completion, 2019, vol. 34, Iss. 3, pp. 171-180.

72. Kelessidis V.C., Dalamarinis P., Maglione R. Experimental study and predictions of pressure losses of fluids modeled as HerschelBulkley in concentric and eccentric annuli in laminar, transitional and turbulent flows. Journal of Petroleum Science and Engineering, 2011, vol. 77, no. 3-4, pp. 305-312.

73. Heydari O., Sahraei E., Skalle P. Investigating the impact of drillpipe's rotation and eccentricity on cuttings transport phenomenon in various horizontal annuluses using computational fluid dynamics (CFD). Journal of Petroleum Science and Engineering, 2017 vol. 156, pp. 801-813.

74. Duan M., Miska S., Yu M., Takach N.E., Ahmed R.M., Hallman J.H. Transport of small cuttings in Exended Reach Drilling. SPE Oil and Gas Conference and Exhibition. Beijing, China, December 2006. pp. 1-9.

75. Gidaspow D. Multiphase flow and fluidization: continuum and $k i-$ netic theory descriptions. London, UK, Academic press, 1994. $461 \mathrm{p}$.

76. Chen Zhu. Experimental study on cuttings transport with foam under simulated horizontal downhole conditions. SPE Drilling \& Completion, 2007, vol. 22, no. 4, pp. 304-312.

77. Duan M., Miska S., Yu M., Takach N.E., Ahmed R.M., Hallman J.H Experimental study and modeling of cuttings transport using foam with drillpipe rotation. SPE Drilling \& completion, 2010, vol. 25, no. 3, pp. 352-362.

78. Ignatenko Y., Bocharov O., Gavrilov A., May R. Steady-state cuttings transport simulation in horizontal borehole annulus. ASME $201837^{\text {th }}$ International Conference on Ocean, Offshore and Arctic Engineering. Madrid, Spain, June 2018, pp. 26-38.

79. Miller R.M., Morris, J.F. Normal stress driven migration and axial development in pressure-driven flow of concentrated suspensions. Journal of Non-Newtonian Fluid Mechanics, 2006, vol. 135, pp. 149-165.

80. Johnson P.C., Jackson R. Frictional collisional constitutive relations for granular materials with application of plane shearing. Journal of Fluid Mechanics, 1987, vol. 176, no. 3, pp. 67-93.

81. Pang B., Wang S., Jiang X., Lu H. Effect of orbital motion of drill pipe on the transport of non-Newtonian fluid-cuttings mixture in horizontal drilling annulus. Journal of Petroleum Science and Engineering, 2019, vol. 174, pp. 201-215.

82. Miska S., Reed T., Kuru E. Advanced cuttings transport study: final technical report. (date of issue: September 30, 2004. DOE Award number: DE-FG26-99 BC15178. The University of Tulsa, 600 South College Avenue, Tulsa, Oklahoma 74104). Tulsa, Oklahoma, 2004. $284 \mathrm{p}$.

83. Busch A., Johansen S.T. Cuttings transport: on the effect of drill pipe rotation and lateral motion on the cuttings bed. Journal of Petroleum Science and Engineering, 2020, vol. 191, pp. 107-136.

84. Pang B., Wang S., Lu C., Cai W., Jiang X., Lu H. Investigation of cuttings transport in directional and horizontal drilling wellbores injected with pulsed drilling fluid using CFD approach. Tunnelling and Underground Space Technology, 2019, vol. 90, pp. 183-193.

85. Shao B., Yan Y., Wang X., Liao F., Yan X. Numerical investigation of a double-circulation system for cuttings transport in CBM well drilling using a CFD-DEM coupled model. Engineering Applications of Computational Fluid Mechanics, 2020, vol. 14, no. 1, pp. 38-52.

86. Al-Gailani A., Al-Yasiri M., Wen D. Numerical study of cuttings transport of nanoparticle-based drilling fluid. Engineering Reports, 2020, no. 2 (e), pp. 12154-12168.

87. Escudier M.P., Nickson A.K., Poole R.J. Turbulent flow of viscoelastic shear-thinning liquids through a rectangular duct: quantification of turbulence anisotropy. Journal of Non-Newtonian Fluid Mechanics, 2009, vol. 160, pp. 2-10.

88. Huque M.M., Butt S., Zendehboudi S., Imtiaz S. Systematic sensitivity analysis of cuttings transport in drilling operation using computational fluid dynamics approach. Journal of Natural Gas Science and Engineering, 2020, vol. 81, no. 103386, pp. 17-38.

89. Zhang F., Wang Y., Wang Y., Miska S., Yu M. Modeling of dynamic cuttings transportation during drilling of oil and gas wells by combining 2D CFD and 1D discretization approach. SPE Journal, 2020, vol. 25, Iss. 3, no. 6, pp. 21-42.

Received: 19 April 2021.

\section{Information about the authors}

Sergey N. Kharlamov, Dr. Sc., professor, National Research Tomsk Polytechnic University.

Janghorbani Mehran, post-graduate student, National Research Tomsk Polytechnic University.

Konstantin A. Filippov, post-graduate student, National Research Tomsk Polytechnic University. 